\title{
AVIFAUNA DE SITIOS ASOCIADOS A LA SELVA TROPICAL EN LA DEPRESIÓN CENTRAL DE CHIAPAS, MÉXICO
}

\author{
Jorge E. RAMÍREZ-ALbORES \\ Facultad de Estudios Superiores Zaragoza Campus II, Universidad Nacional Autónoma de México. \\ MÉXICO, D. F. E-mail: jorgeramirez22@hotmail.com
}

Ramírez-Albores, J. E. 2010. Avifauna de sitios asociados a la selva tropical en la Depresión Central de Chiapas, México. Acta Zool. Mex. (n. s.), 26(3): 539-562.

RESUMEN. Se realizó una descripción de la composición y estructura de la avifauna de sitios asociados a la selva tropical en la Depresión Central de Chiapas, de 2003 a 2004. La riqueza avifaunística en el área de estudio fue de 364 especies considerando el trabajo de campo y los registros previos provenientes de literatura y de especímenes depositados en colecciones. Las familias con mayor riqueza de especies fueron Tyrannidae, Parulidae, Icteridae y Trochilidae. El componente estacional de las especies indica que el 68\% son residentes y el $32 \%$ restante son migratorias. En cuanto a su abundancia, la mayoría de las especies son consideradas como raras (34\%), mientras que las especies comunes y abundantes constituyen el $11 \%$ y $3 \%$ del total, respectivamente. La selva baja caducifolia registró el mayor número de especies (226), seguida por la vegetación secundaria (163) y la selva mediana subperennifolia (122). De las especies registradas, 136 son registros adicionales para la región de estudio y 56 para el Parque Nacional Cañón del Sumidero. La avifauna registrada incluye el $45 \%$ de la avifauna estatal, lo que destaca la relevancia de esta región y una urgente necesidad de establecer estrategias de manejo para su conservación y protección. La cantidad de especies incrementadas y la falta de información en la región sugieren la necesidad de inventarios locales y regionales para un conocimiento más preciso sobre la distribución de las especies.

Palabras clave: Aves, riqueza de especies, selva baja caducifolia, Cañón del Sumidero, Chiapas.

Ramírez-Albores, J. E. 2010. Birds of associated sites to tropical forest in the Chiapas's Central Depression, Mexico. Acta Zool. Mex. (n. s.), 26(3): 539-562.

ABSTRACT. This paper describes the birds' structure and composition of associated sites to tropical forest in the Central Depression of Chiapas, Mexico, through 2003 to 2004. I recorded 364 species considering scientific literature, museum collections and field work. Families with highest species richness were Tyrannidae, Parulidae, Icteridae and Trochilidae. Resident birds represented $68 \%$ of the total and the $32 \%$ remaining were migratory. Thirty four percent of bird species were rare, $11 \%$ common and 3\% abundant. Highest species richness was found for the tropical deciduous forest (226), followed by the secondary forest (163) and the tropical semideciduous forest (122). From all birds, 136 were additional records in the region and, 56 were for the Sumidero Canyon National Park. The species richness recorded included $45 \%$ of the avifauna of Chiapas, indicating the relevance of this region and the urgent need to establish conservation and protection management strategies. The magnitude of species increment and lack of information in this region suggest that regional and local inventories are necessaries to improve the knowledge about species distribution.

Key words: Birds, species richness, tropical deciduous forest, Sumidero Canyon, Chiapas.

Recibido: 19/11/2009; aceptado: 25/05/2010. 


\section{INTRODUCCIÓN}

La diversidad biológica se distribuye de manera heterogénea en el mundo. La distribución diferencial de la diversidad biológica y sus patrones son resultado de numerosos factores y procesos locales y regionales, tales como factores históricos, evolutivos, geográficos, ambientales, estocásticos, ecológicos y recientemente, de las modificaciones en el uso del suelo y los diversos sistemas productivos utilizados por el hombre que determinan esta heterogeneidad (Rickelfs \& Schluter 1993, Gaston 2000, Whittaker et al. 2001). Sin embargo, procesos como la pérdida, degradación y fragmentación de los ambientes naturales modifican los patrones de distribución, abundancia y composición de especies de las comunidades en prácticamente todos los ambientes (Rangel-Salazar et al. 2009). En México grandes extensiones de bosque y selvas tropicales han experimentado cambios dramáticos por el establecimiento de pastizales y zonas de cultivo (Challenger 1998), es posible que estas modificaciones hayan precipitado cambios en la composición y diversidad de especies (Ceballos \& García 1995, Trejo 1998).

La Depresión Central de Chiapas se extiende paralelamente al noreste de la Sierra Madre de Chiapas, atraviesa totalmente el estado de Chiapas y el extremo oriental de Oaxaca y comprende una extensión de aprox. 9,000 $\mathrm{Km}^{2}$ (Mülleried 1957). Tiene una alta importancia por su biodiversidad, ya que se encuentran áreas naturales como el Parque Nacional Cañón del Sumidero, la Región Terrestre Prioritaria para la Conservación (RTP-141 La Chacona-Cañón del Sumidero; Arriaga et al. 2000), la Reserva Ecológica El Zapotal-Mactumatza, Áreas de Importancia para la Conservación de las Aves en México (AICA's) como el Corredor Laguna BélgicaSierra Limón-Cañón del Sumidero (AICA-46) y el Zapotal-Mactumatza (AICA-54; Arizmendi \& Márquez 2000) y reconocida como un humedal por la Convención RAMSAR, además, de encontrarse inmersa dentro del Corredor Biológico Mesoamericano. Está región se caracteriza por presentar áreas de selva baja caducifolia y selva mediana subperennifolia (Miranda 1975). Sin embargo, debido al intenso crecimiento demográfico en años recientes, los principales problemas de conservación que enfrenta esta región están relacionados con la expansión de la frontera agropecuaria, los incendios forestales, la extracción de leña, la contaminación de cuerpos y corrientes de agua y la ganadería, al quedar algunos relictos de selva tropical lo que conlleva a la pérdida de la biodiversidad presente en esta área (Reyes \& Souza 1997, Challenger 1998). Aunado a esto, los estudios ornitológicos que se han realizado en esta región son escasos y no se tenía reporte de ningún trabajo de investigación que arrojara un número confiable de especies, o están orientados al registro de presencia de especies (Álvarez del Toro 1948, 1952, 1954; Ovando 1990, Fernández 2002, Altamirano 2007), a relaciones con el paisaje a escala local (Ramírez-Albores 2004, 2010) y a las listas que para la designación de las Áreas de Importancia para la Conservación de las Aves en México se han publicado 
(Arizmendi \& Márquez 2000). Recientemente, Rangel-Salazar et al. (2005) mencionan que en esta región se distribuyen 297 especies de aves. Backyard Nature (adaptado de Howell \& Webb 1995) menciona que la riqueza avifaunística potencial para esta región es de 321 especies. Pero estos trabajos infieren o extrapolan la distribución de algunas especies, y no incluyen algunos de los estudios que aquí se presentan. Por este motivo, era necesario actualizar y proporcionar datos recientes de la avifauna presente en esta región para ampliar su conocimiento y proponer medidas de conservación, ya que en el contexto nacional y regional, los estudios y registros avifaunísticos a escalas locales y regionales son de gran aporte para el conocimiento detallado de la distribución geográfica de las especies y su conservación (Navarro \& Sánchez-González 2003, Rojas-Soto \& Oliveras de Ita 2005). Por lo anterior, el propósito de este estudio es describir detalladamente la composición y estructura de la avifauna de sitios asociados a la selva tropical en la Depresión Central de Chiapas, como una forma de incrementar el conocimiento que se tiene de la avifauna en esta región y como información base para estudios posteriores.

\section{MATERIAL Y MÉTODOS}

El estudio se llevó a cabo en sitios ubicados en los municipios de San Fernando (14 sitios entre $16^{\circ} 52^{\prime} \mathrm{N}$ y $93^{\circ} 12^{\prime} \mathrm{O}$, y de 600 a $\left.1200 \mathrm{msnm}\right)$, Tuxtla Gutiérrez $\left(16^{\circ} 45^{\prime} \mathrm{N}\right.$, $93^{\circ} 06^{\prime} \mathrm{O} ; 16^{\circ} 45^{\prime} \mathrm{N}, 93^{\circ} 08^{\prime} \mathrm{O} ; 16^{\circ} 45^{\prime} \mathrm{N}, 93^{\circ} 05^{\prime} \mathrm{O} ; 16^{\circ} 48^{\prime} \mathrm{N}, 93^{\circ} 07^{\prime} \mathrm{O}$; entre 500 a 810 msnm), Chiapa de Corzo $\left(16^{\circ} 42^{\prime} \mathrm{N}, 93^{\circ} 01^{\prime} \mathrm{O} ; 16^{\circ} 41^{\prime} \mathrm{N}, 92^{\circ} 59^{\prime} \mathrm{O}\right.$; entre 440 a 490 $\mathrm{msnm})$, Osumacinta $\left(16^{\circ} 56^{\prime} \mathrm{N}, 93^{\circ} 05^{\prime} \mathrm{O} ; 16^{\circ} 55^{\prime} \mathrm{N}, 93^{\circ} 04^{\prime} \mathrm{O}\right.$; entre 590 a $\left.635 \mathrm{msnm}\right)$ y Chicoasén $\left(16^{\circ} 57^{\prime} \mathrm{N}, 93^{\circ} 08^{\prime} \mathrm{O} ; 16^{\circ} 58^{\prime} \mathrm{N}, 93^{\circ} 10^{\prime} \mathrm{O}\right.$; entre 590 a $\left.930 \mathrm{msnm}\right)$, localizados en la Depresión Central de Chiapas (Fig. 1). El clima va de cálido subhúmedo con lluvias en verano (de mayo a octubre, siendo mayo el mes más caluroso) a semicálido subhúmedo en altitudes superiores a los $1,000 \mathrm{msnm}$, la temperatura media anual es de $24^{\circ} \mathrm{C}$ y la precipitación anual varía entre 500 a 2,500 mm (FORTAM 1984; INEGI 2004, 2006). El principal tipo de vegetación en el área de estudio es la selva baja caducifolia, asociado a una mezcla de manchones de selva mediana subperennifolia, encinar tropical y vegetación riparia, además, de zonas con distinto grado de sucesión vegetal, cultivos, pastizales/potreros, zonas urbanas y suburbanas (Miranda 1975, Reyes \& Souza 1997). Las especies arbóreas dominantes en el área son: Bursera simaruba, B. bipinnata, Casearia sp., Ceiba sp., Guettarda sp., Ardisia sp., Alvaradoa amorphoides, Jaquinia laurantifolia, Ficus cookii, Cedrela odorata, Daphnopsis mexicana y Acacia cornigera (Reyes \& Souza 1997).

El trabajo de campo consistió en 24 visitas mensuales, sumando un total de 288 días, de febrero de 2003 a noviembre de 2004. Se empleó el método de conteo por puntos de radio fijo de $25 \mathrm{~m}$ (Hutto et al.1986, Ralph et al. 1995). En estos puntos se registraron todas las especies de aves observadas y escuchadas durante un periodo de 5 minutos, y con una distancia mínima aproximada de $100 \mathrm{~m}$ entre cada punto (Hutto et al.1986). 


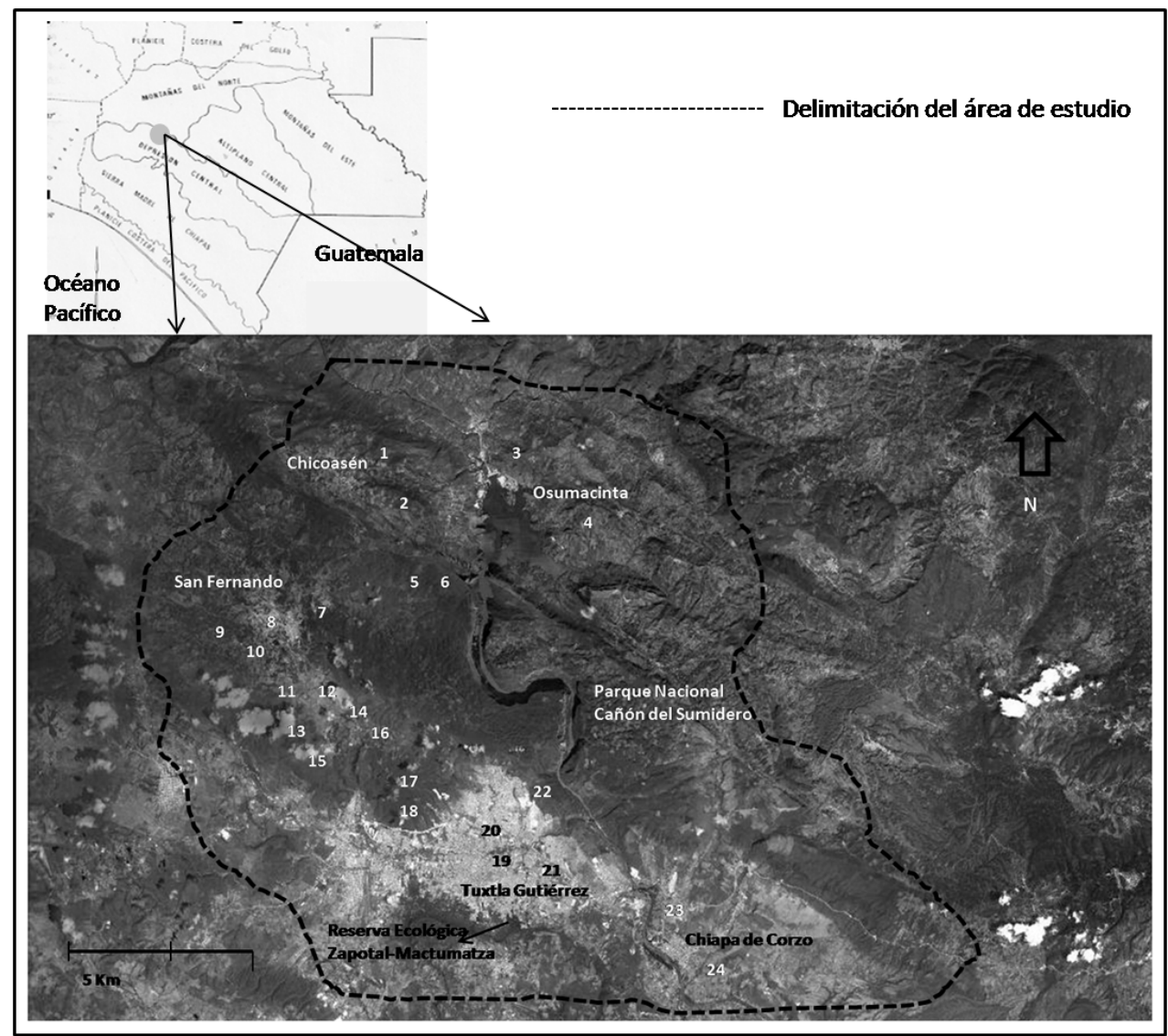

Figura 1. Localización de los sitios de estudio en la Depresión Central de Chiapas, Chiapas.

Los sitios de muestreo fueron visitados una vez al mes y se ubicaron aleatoriamente. La riqueza de especies en los sitios de estudio y por tipo de vegetación se obtuvo al sumar las especies observadas y escuchadas, así como las especies observadas en la periferia de estos.

Las observaciones y registros auditivos se llevaron a cabo entre las 7:00-11:00 hrs. y de 16:00-19:00 hrs. con ayuda de binoculares $\left(10\right.$ x 50 KONUS $\left.{ }^{\circledR} 2104\right)$ y guías de campo (Peterson \& Chalif 1989, Howell \& Webb 1995, National Geographic Society 1999). Los registros auditivos de las especies grabados en campo fueron cotejados con la Fonoteca de las Aves de Chiapas (Duncan 1993). La nomenclatura y secuencia taxonómica de las especies registradas fue basada en el arreglo sistemático de la Unión Americana de Ornitólogos (AOU 2008). Adicionalmente, se 
recopiló información de trabajos previos publicados, inéditos, tesis y en extrapolaciones realizados en el área de estudio (Álvarez del Toro 1948, 1952, 1954; Ovando 1990, Gómez de Silva et al. 1999, Morales et al. 1999, Fernández 2002, Altamirano 2004, 2007; Ramírez-Albores 2004, 2010; Rangel-Salazar et al. 2005), así como los registros contenidos en el Atlas de las Aves de México (Navarro et al. 2003). Además, de corroborar la información de su distribución (de acuerdo con Howell \& Webb 1995) y de confirmar en campo registros que habían sido propuestos como de posible ocurrencia, se consultaron las bases de datos de museos y colecciones disponibles: Unidad de Informática para la Biodiversidad (UNIBIOCNAV, www.unibio.ibunam.unam.mx, enero de 2009), Red Mundial de Información sobre Biodiversidad (REMIB, www.conabio.gob.mx, enero de 2009), Ornis (http://olla.berkeley.edu/ornisnet, febrero de 2009), Integrated Taxonomic Information System (ITIS, www.itis.gov, febrero de 2009) y Global Biodiversity Information Facility (GBIF, www.gbif.org, febrero de 2009).

La ocurrencia estacional de las especies se basó en las observaciones en campo y en la propuesta por Howell y Webb (1995): residente, residente de verano, transitoria, ocasional y visitante de invierno. La abundancia relativa de las especies se determinó dividiendo el número de individuos de la especie ${ }_{i}$ entre el número total de individuos, multiplicado por 100 para obtener el porcentaje $\left(P_{i}=n \mathrm{~s} / \Sigma n\right.$, donde $P_{i}$ representa la abundancia relativa de la especie ${ }_{i}, n \mathrm{~s}$ el número de individuos de la especie ${ }_{i}, \Sigma n$ el número total de individuos; Pettingil 1969). Las categorías de abundancia relativa fueron: abundante (90-100\%), común (65-89\%), moderadamente común (31-64\%), no común (10-30\%) y rara (1-9\%). El endemismo se determinó con base a Navarro y Benítez (1993), Howell y Webb (1995) y González-García y Gómez de Silva (2003). El estatus de conservación de las especies se basó en la Norma Oficial Mexicana (NOM-059-SEMARNAT-2001; DOF 2002) y IUCN (2009).

Para comparar la riqueza de especies entre los diferentes tipos de vegetación se utilizó una prueba de $x^{2}$, considerando los análisis estadísticamente significativos cuando $P<0.05$. El análisis sobre abundancia relativa y distribución por tipo de vegetación se aplicó sólo a las especies registradas durante el trabajo de campo, y aquellos registros obtenidos de la literatura y/o de alguna colección consultada fueron descartados.

\section{RESULTADOS}

Las observaciones de campo registraron 278 especies de aves, que aunado con los registros provenientes de colecciones y/o literatura elevan la riqueza de la región de estudio a 364 especies, pertenecientes a 53 familias y 18 órdenes (Apéndice 1). Las familias con más especies fueron Tyrannidae y Parulidae con 38 especies, seguida de las familias Trochilidae con 23, Icteridae con 17, y Accipitridae con 15 (Apéndice 1). Estas familias en conjunto incluyeron el $36 \%$ del total de especies registradas. 
La ocurrencia estacional se dividió en, 249 especies (68\%) residentes, $81(22 \%)$ visitantes de invierno, $28(8 \%)$ transitorias, cuatro $(1 \%)$ residentes de verano y una (1\%) ocasional (Apéndice 1). Mientras que la abundancia relativa fue dividida en, 94 especies raras, 77 no comunes, 67 moderadamente comunes, 32 comunes y ocho abundantes (Apéndice 1). En la distribución por tipo de vegetación, la mayor riqueza de especies fue registrada en la selva baja caducifolia (226), seguida por la vegetación secundaria (163) y selva mediana subperennifolia (122), y la menor riqueza de especies fue en la vegetación riparia (54), encinar tropical (51), y las especies con hábitos acuáticos y subacuáticos (23; Fig. 2, Apéndice 1).

Del total de especies registradas (278 especies), 20 se localizaron exclusivamente en la selva baja caducifolia, cuatro en la selva mediana subperennifolia, una en los potreros/pastizales, dos en la zona urbana y suburbana (Apéndice 1). El 76\% (172 especies) y el $74 \%$ (91 especies) de la avifauna es residente de la selva baja caducifolia y la selva mediana subperennifolia, respectivamente y el resto correspondio a las migratorias (Fig. 2). En el encinar tropical, en las zonas de cultivo y en los potreros/pastizales se registró el 72\% (37 especies), 75\% (49 especies) y $77 \%$ (48 especies) respectivamente, de las especies residentes (Fig. 2). En contraste con los resultados anteriores, la proporción de especies migratorias fue más alta con respecto a las especies que se distribuyen en ambientes acuáticos o subacuáticos (56\%, 13 especies; Fig. 2). Al analizar comparativamente los tipos de vegetación por riqueza de especies, no se encontraron diferencias significativas entre la selva baja caducifolia, selva mediana subperennifolia y vegetación secundaria $\left(\chi^{2}=3.45\right.$, gl. $=$ 43, $P=0.75)$, y entre encinar tropical, cercos vivos, cultivos, vegetación riparia, pastizales/potreros, y zonas urbanas y suburbanas $\left(\chi^{2}=2.28, \mathrm{gl} .=61, P=0.66\right)$. Pero se encontraron diferencias significativas en la riqueza de especies entre la selva baja caducifolia, selva mediana subperennifolia y vegetación secundaria con los demás tipos de vegetación $\left(\chi^{2}=22.35, \mathrm{gl} .=75, P=0.04\right)$.

En cuanto al estatus de conservación, 14 especies se encuentran amenazadas (e.g. Penelope purpurascens, Crax rubra), 40 bajo protección especial, y seis de acuerdo a la IUCN: cuatro casi amenazadas (Colinus virginianus, Cyrtonyx ocellatus, Megascops barbarus, Xenotriccus callizonus) y dos vulnerables (Penelopina nigra, Crax rubra). Las especies Aratinga holochlora, Megascops barbarus, Amazilia viridifrons, A. yucatanensis, Momotus mexicanus, Xenotriccus callizonus, Deltarhynchus flammulatus, Uropsila leucogastra, Ptilogonys cinereus, Basileuterus rufifrons, Granatellus venustus, Arremonops rufivirgatus, Pheucticus chrysopeplus y Cacicus melanicterus son endémicas o cuasiendémicas a México, lo que representa el $4 \%$ de las aves endémicas del país (Navarro \& Benítez 1993, González-García \& Gómez de Silva 2003 ) y el $40 \%$ del total de especies endémicas registradas para Chiapas (RangelSalazar et al. 2005). De acuerdo a la propuesta de Ríos (2006), en la zona de estudio se registraron 86 especies de las 337 endémicas a Mesoamérica (e.g. Panyptila 


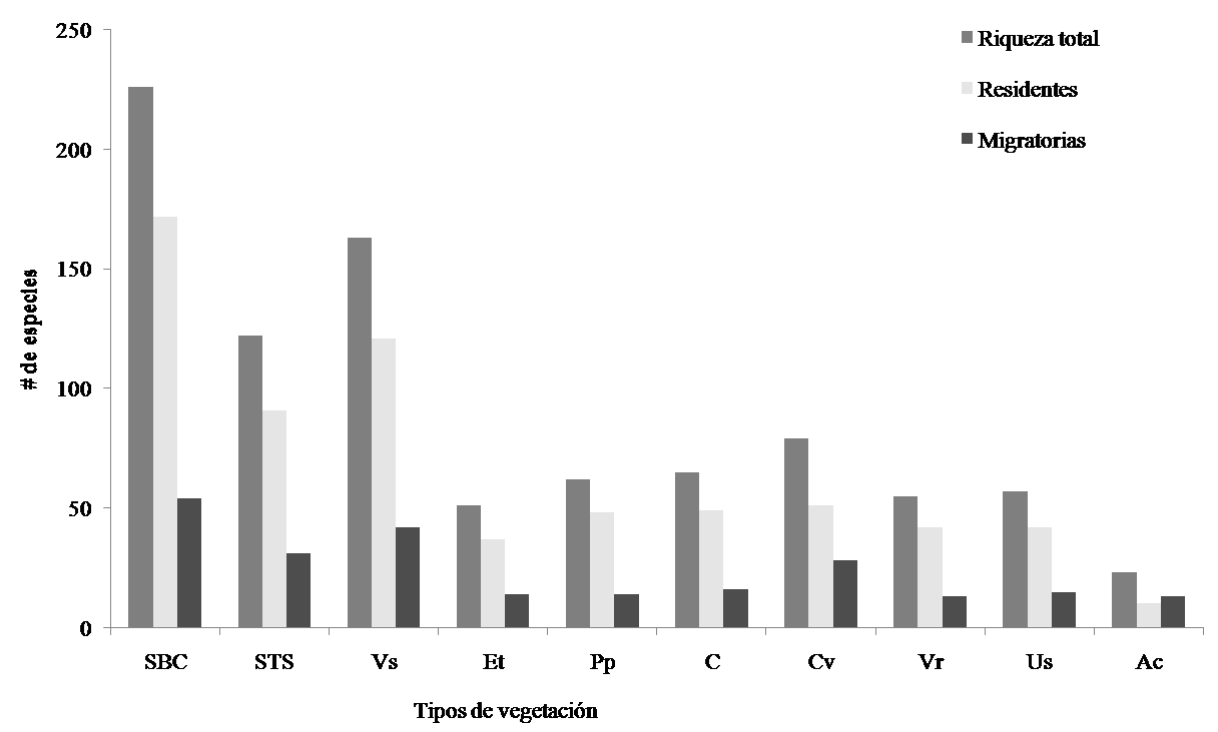

Figura 2. Riqueza de especies en los diferentes tipos de vegetación (SBC, selva baja caducifolia; Pp, pastizal/potrero; $\mathrm{Cv}$, cercos vivos; Vr, vegetación riparia; STS, selva mediana subperennifolia; Vs, vegetación secundaria; C, cultivo; Et, encinar tropical; Us, zona urbana y suburbana, incluye parques y centros de recreación; Ac, acuáticas y subacuáticas).

sanctihieronymi, Rhynchocynclus brevirostris), seis de las 84 endémicas al oeste de México (e.g. Granatellus venustus, Icterus pustulatus) y 92 de las 278 asociadas a la selva baja caducifolia (e.g. Florigusa mellivora, Cyanocorax morio; Apéndice 1).

Como resultado de este estudio, se obtuvieron 136 registros adicionales para la Depresión Central de Chiapas (Apéndice 1): 29 provenientes de observaciones de campo durante la realización del presente estudio (e.g. Trogon melanocephalus, Eucomites penicillata), 31 provenientes de colecciones y/o literatura no corroborados en campo (e.g. Penelopina nigra, Megascops barbarus), 34 provenientes de colecciones y/o literatura cuyos registros fueron corroborados en campo (e.g. Rostrhamus sociabilis, Psarocolius wagleri) y 44 provenientes de observaciones de campo y de colecciones y/o literatura (e.g. Chondrohierax uncinatus, Campephilus guatemalensis), que de acuerdo a Howell y Webb (1995) se distribuyen en esta región pero no son considerados por Rangel-Salazar et al. (2005). Además, de 56 registros adicionales para el Parque Nacional Cañón del Sumidero (Apéndice 1): 49 provenientes de observaciones de campo durante la realización del presente estudio (e.g. Crypturellus soui, Pulsatrix perspicillata), y siete provenientes de colecciones y/o literatura no corroborados en campo (e.g., Crax rubra, Pelecanus erythrorhynchos). 


\section{DISCUSIÓN}

En este estudio se documentó la presencia de 433 especies de aves para la Depresión Central de Chiapas, lo que equivale al $66 \%$ de las aves registradas en Chiapas (Álvarez del Toro 1980, Palomera et al. 1995, Rangel-Salazar et al. 2005) y el 41\% de las aves de México (Arizmendi \& Márquez 2000). Lo cual es notable si se considera que está región representa aproximadamente el $12 \%$ de la superficie estatal. La Depresión Central de Chiapas es de gran importancia para la avifauna como lugar de paso para las especies migratorias, por la presencia de un número significativo de endemismos y por la compleja topografía e historia biogeografica que ha permitido la existencia de varios tipos de vegetación que inciden en la diversidad de aves (Escalante et al. 1993, Arriaga et al. 2000). Sin embargo, pocos son los estudios que se han desarrollado en esta región, que en su mayoría se han llevado a cabo en la Selva Lacandona, El Triunfo, el Soconusco, los Altos de Chiapas y la Planicie Costera del Pacífico (Rangel-Salazar et al. 2005). Además, aunado a lo anterior, la acentuada pérdida de la cobertura vegetal debido a las presiones antropogénicas (Miranda 1975, Reyes \& Souza 1997), podría representar cambios o reducciones considerables en la distribución de algunas especies de aves. Por lo que, el inventario obtenido podría considerarse útil y representativo para la avifauna de la Depresión Central de Chiapas, en la que se adicionaron y fueron confirmados registros de especies cuya distribución había sido inferida (e.g. Rangel-Salazar et al. 2005), ya que puede servir de referencia para futuras investigaciones que se realicen en la región.

Con base en el Atlas de la Aves de México (Navarro et al. 2003) y otra literatura (Álvarez del Toro 1948, 1952, 1954; Ovando 1990, Gómez de Silva et al. 1999, Morales et al. 1999, Fernández 2002, Altamirano 2004, 2007; Ramírez-Albores 2004, 2010; Rangel-Salazar et al. 2005), se lograron compilar 364 registros de especies para el área de estudio, de las cuales se confirmó la presencia en campo de 278. Los registros de las especies que no fueron corroborados en campo corresponden principalmente a especies migratorias: de ambientes acuáticos o subacuáticos (e.g. Porzana carolina, Calidris melanotus), de presencia ocasional (e.g. Buteo platypterus, Leucophaeus pipixcan), de ambientes montanos (e.g. Vermivora peregrina), de hábitos nocturnos (e.g. Chordeiles minor, Caprimulgus carolinensis) o registros históricos pero no actuales en el área de estudio (e.g. Pelecanus erythrorhynchos); y residentes: de ambientes acuáticos o subacuáticos (e.g. Egretta tricolor, Fulica americana), de ambientes montanos (e.g. Cyrtonyx ocellatus, Myioborus miniatus), de hábitos nocturnos (e.g. Megascops trichopsis, Nyctibius jamaicensis) o registros históricos pero no actuales en el área de estudio (e.g. Crax rubra). Lo anterior sugiere que este tipo de especies amerita un mayor esfuerzo de muestreo para complementar el listado obtenido. 
El presente listado comparte 227 especies con lo reportado por Rangel-Salazar et al. (2005). Sin embargo, aunque estos últimos autores mencionan 113 especies que no fueron reportadas en este estudio, no mencionan 136 especies aquí reportadas que son nuevas adiciones al listado anterior propuesto para la Depresión Central de Chiapas (Backyard Nature adaptado de Howell \& Webb 1995, Rangel-Salazar et al. 2005), lo que aumento el conocimiento de la riqueza avifaunística en un $45 \%$ de esta región (433 especies de aves). Además, se realizaron 56 nuevas adiciones para el Parque Nacional Cañón del Sumidero, lo que aumentó la riqueza avifaunística en un $22 \%$, para un total de 302 especies que se distribuyen en esta área natural.

En los sitios de estudio es acentuada la pérdida de la cobertura vegetal debido a la expansión de la frontera agropecuaria, los incendios forestales, la extracción de leña, la contaminación de cuerpos y corrientes de agua y la ganadería (Miranda 1975, Reyes \& Souza 1997), que podría representar la disminución de la disponibilidad de recursos para gran parte de especies de aves. Sin embargo, para determinar que tan completo es un inventario serían necesarios criterios adicionales, tales como las curvas de acumulación, la comparación del número de especies registradas en zonas con el mismo gradiente altitudinal y/o tipos de vegetación. Por otro lado, la Depresión Central de Chiapas es el punto de contacto de varios ambientes (e.g. selva baja caducifolia, selva mediana subperennifolia y pequeños manchones de encinar tropical), es de gran importancia como lugar de paso para las especies que migran hacia el sur y/o Golfo de México y Océano Pacífico y por la compleja topografía e historia biogeografica que ha permitido la existencia de varios tipos de vegetación que inciden en la diversidad de aves (Escalante et al. 1993, Arriaga et al. 2000). Además, es una región que tiene una gran importancia para la avifauna de México, ya que constituye una región donde se mezclan especies endémicas del Pacífico seco (e.g. Piaya cayana, Amazilia viridifrons), especies tropicales centroamericanas (e.g. Crypturellus cinnamomeus, Synallaxis erythrothorax) con las propias del área como Xenotriccus callizonus (Navarro \& Peterson 2004, Ríos 2006). En esta región existen especies como Amazilia viridifrons, Deltharhynchus flammulatus, Granatellus venustus, Sporophila torqueola, Pheucticus chrysopeplus e Icterus pustulatus que se distribuyen a lo largo de la costa del Pacífico mexicano, pero entran a la Depresión Central de Chiapas por el Istmo de Tehuantepec siguiendo un continuo de tierras bajas y alejándose de la costa de Chiapas para introducirse en el centro del estado (Ríos 2006).

Es posible que para la zona de estudio, los movimientos altitudinales y latitudinales estén correlacionados con las fluctuaciones en la abundancia de las especies (Arizmendi et al. 1990, Moya-Moreno 2002); por ejemplo, algunas de las especies raras son claramente características de ambientes templados cuyas poblaciones se dispersan a otros sitios durante épocas de escasez de recursos (e.g. Tilmatura dupontii, Dendroica townsendi), o son migratorias de paso que se 
presentan en pocas cantidades en el interior de Chiapas (e.g. Dendroica fusca, Wilsonia canadensis). Sin embargo, estos cambios estacionales en la abundancia, posiblemente asociados también a la fenología estacional de la selva baja caducifolia, no se evaluaron, por lo que necesitaran ser analizados con detalle posteriormente. La mayor riqueza de especies por tipo de vegetación se encontró en la selva baja caducifolia, quizá debido a que la gran complejidad de su estructura lo hace uno de los ambientes más ricos en México (Ceballos \& García 1995, Trejo 1998). Estos resultados pueden ser atribuibles a la existencia de una mayor diversidad florística en este tipo de vegetación (Trejo 1998) que se traduce a su vez en mayor disponibilidad de recursos (Arizmendi et al. 1990, Ortiz-Pulido et al. 1995, Ramírez-Albores \& Ramírez 2002, Almazán-Núñez \& Navarro 2006).

La distribución de la avifauna en las diferentes regiones fisiográficas de Chiapas es altamente heterogénea (Rangel-Salazar et al. 2005), y es posible que ocurra también heterogeneidad al interior de cada región, o inclusive entre regiones fisiográficas contiguas (González-Domínguez 1998). Por lo que el comportamiento de la avifauna de la Depresión Central de Chiapas puede ser compartida o influenciada por otras regiones, como las Montañas del Oriente, la Altiplanicie Central e inclusive por la Sierra Madre de Chiapas, lo que le confiere la capacidad de albergar especies de estas regiones (e.g. Thryothorus maculipectus, Icterus maculialatus; Altamirano 2004).

Los inventarios avifaunísticos aportan evidencia de la importancia de una región, sobre todo de áreas que no han sido estudiadas y/o que presentan alteraciones importantes en sus ecosistemas, como es el caso de la Depresión Central de Chiapas que como muchas otras regiones del país, está siendo afectada por diversos factores como la expansión de la frontera agropecuaria, los incendios forestales, el crecimiento demográfico y la ganadería. La carencia de estudios avifaunísticos repercute en la delimitación actual de las áreas de distribución y por ende puede limitar el planteamiento de acciones de conservación, por lo que es necesario que se continúen realizando inventarios locales y/o regionales para incrementar el conocimiento, necesidades y oportunidades de conservación de la avifauna (Navarro \& Sánchez-González 2003, Rojas-Soto \& Oliveras de Ita 2005).

El aporte principal del presente estudio fue la compilación de la información avifaunística de la región de la Depresión Central de Chiapas. De esta manera el inventario obtenido podría considerarse útil y representativo para la avifauna de esta región, en la que se adicionaron y fueron confirmados registros de especies cuya distribución potencial había sido inferida (Howell \& Webb 1995, Rangel-Salazar et al. 2005), lo que aumento el conocimiento de la riqueza avifaunística en un 45\% (433 especies de aves). Además, de que puede servir de referencia para futuras investigaciones que se realicen en la región. 
AGRADECIMIENTOS. A Adolfo Navarro por sus valiosos comentarios y sugerencias realizados a este manuscrito. Al Colegio de la Frontera Sur y al CONACyT por las becas otorgadas. Al Museo de Zoología "Alfonso L. Herrera" de la Facultad de Ciencias, UNAM por su apoyo en la consulta de la base del Atlas de las Aves de México, así como a Fanny Rebón por permitirme el acceso a la colección de aves de esté museo. A Patricia Escalante por permitirme el acceso a la Colección Nacional de Aves del Instituto de Biología, UNAM. Agradezco a todas las personas que colaboraron directa o indirectamente en el desarrollo de este estudio y a los valiosos comentarios y sugerencias de los revisores anónimos.

\section{LITERATURA CITADA}

Almazán-Nuñez, R.C. \& A.G. Navarro S. 2006. Avifauna de la subcuenca del río San Juan, Guerrero, México. Revista Mexicana de Biodiversidad, 77: 103-114.

Altamirano G.O., M.A. 2004. Obtención de la riqueza de aves y selección de especies susceptibles de monitoreo en la zona noroeste en el estado de Chiapas. Instituto de Historia Natural y Ecología. Informe final SNIB-Conabio proyecto No. Y018, México, D.F.

Altamirano G.O., M.A. 2007. Vertebrados terrestres del Parque Nacional Cañón del Sumidero, Chiapas, México. Instituto de Historia Natural y Ecología. Informe final SNIB-Conabio proyecto No. BK003, México, D.F.

Álvarez del Toro, M. 1948. The White Pelican in the interior of Chiapas, Mexico. Auk, 65: 457-458.

Álvarez del Toro, M. 1952. New records of birds from Chiapas, Mexico. Condor, 54: 112-114.

Álvarez del Toro, M. 1954. Notes on the occurrence of birds in Chiapas, Mexico. Auk, 56: 365.

Álvarez del Toro, M. 1980. Las aves de Chiapas. 2a. ed. Gobierno del Estado de Chiapas. Tuxtla Gutiérrez, Chiapas, México.

American Ornithologist' Union (AOU). 2008. Check-list of North American birds. 7th edition. http://www.aou.org.

Arizmendi, M.C. \& L. Márquez. 2000. Áreas de importancia para la conservación de las aves en México. Cipamex. México.

Arizmendi, M.C., H. Berlanga, L. Márquez, L. Navarijo \& J.F. Ornelas. 1990. Avifauna de la región de Chamela, Jalisco. Cuadernos 4. Instituto de Biología, UNAM. México, D.F.

Arriaga, L., J., M. Espinoza, C. Aguilar, E. Martínez, L. Gómez, E. Loa \& J. Larson. 2000. Regiones prioritarias terrestres de México. Conabio. México, D.F.

Ceballos, G. \& A. García.1995. Conserving neotropical biodiversity: the role of dry forest in western Mexico. Conservation Biology, 9: 1349-1353.

Challenger, A.1998. Utilización y conservación de los ecosistemas terrestres de México. Pasado, presente y futuro. Conabio-Instituto de Biología, UNAM-Sierra Madre. México, D.F.

DOF (Diario Oficial de la Federación). 2002. Norma Oficial Mexicana NOM-059-SEMARNAT-2001. Protección ambiental-especies nativas de México de flora y fauna silvestres-categorías de riesgo y especificaciones para su inclusión, exclusión o cambio-lista de especies en riesgo. México, D.F.

Duncan, C.D. 1993. Manual para el uso de la fonoteca de las aves de Chiapas "cassettes 1-8". Pronatura-Instituto para la Ornitología del Campo, Universidad de Maine en Manchias. ME.

Escalante, P., A.G. Navarro S. \& A.T. Peterson. 1993. A geographic, ecological, and historical analysis of land bird diversity in México, Pp. 281-307. In: T.P. Ramamorthy, R. Bye, A. Lot and J. Fa (Eds.). The biological diversity of Mexico: Origins and distribution. Oxford University Press, New York.

Fernández, M.Y. 2002. Áreas naturales en ciudades y su conservación: El caso de la avifauna de El Zapotal, Tuxtla Gutiérrez, Chiapas, México. Tesis de Maestría. El Colegio de la Frontera Sur. San Cristóbal de las Casas, Chiapas, México. 
FORTAM. 1984. San Fernando, Diagnóstico Municipal. Gobierno Federal-Estatal-Municipal Plan Chiapas. Talleres Gráficos del Estado. Tuxtla Gutiérrez, Chiapas, México.

Gaston, K.J. 2000. Global patterns in biodiversity. Nature, 405: 220-227.

Gómez de Silva, H., E. Morales, G. Cartas, R. Megchón, G. Velasco, F. Gómez \& M. Tuz. 1999. AICA 191: Corredor Laguna Bélgica-Sierra Limón-Cañón del Sumidero. In: H. Benítez, C. Arizmendi and L. Márquez. (eds.). Base de datos de las AICAS. Cipamex, Conabio, FMCN y CCA. (http://www.conabio.gob.mx). México.

González-Domínguez, P. 1998. Análisis avifaunístico en cuatro áreas naturales de Chiapas. Tesis de Licenciatura, Universidad Autónoma de Guadalajara. Guadalajara, Jalisco, México.

González-García, F. \& H. Gómez de Silva. 2003. Especies endémicas: riqueza, patrones de distribución y retos para su conservación. Pp. 150-194. In: H. Gómez de Silva and A. Oliveras de Ita (Eds.). Conservación de aves. Experiencias en México. Cipamex-Conabio-National Fish and Wildlife Foundation. México, D.F.

Howell, S.N. \& S. Webb. 1995. A guide to the birds of Mexico and northern Central America. Oxford University Press. Oxford.

Hutto, R.L., S.M. Pletschet \& P. Hendricks. 1986. A fixed-radius point count method for non-breeding and breeding season use. Auk, 103: 593-602.

INEGI (Instituto Nacional de Estadística y Geografía). 2004. Cuaderno estadístico municipal de Chiapa de Corzo. INEGI. México.

INEGI (Instituto Nacional de Estadística y Geografía). 2006. Cuaderno estadístico municipal de Tuxtla Gutiérrez. INEGI. México.

IUCN (International Union for Conservation of Nature). 2009. IUCN Red list of threatened species. Version 2009.1. http://www.iucnredlist.org. Downloaded on 23 august 2009.

Miranda, F. 1975. La vegetación de Chiapas. $2^{\mathrm{a}}$. ed. Gobierno del Estado. Tuxtla Gutiérrez, Chiapas, México.

Morales, E., J. Rangel, R. Megchón, G. Velasco, G. Cartas, F. Gómez \& M. Tuz. 1999. AICA 199: Zapotal-Mactumaza. In: H. Benítez, C. Arizmendi and L. Márquez. (Eds.). Base de datos de las AICAS. Cipamex, Conabio, FMCN y CCA. (http://www.conabio.gob.mx). México.

Moya-Moreno, H. 2002. Disponibilidad de alimento y estructura del hábitat en la distribución y abundancia de aves insectívoras en una selva baja en Estipac, Jalisco. Tesis de Licenciatura. Facultad de Ciencias, UNAM. México, D.F.

Mülleried, F.K.G. 1957. Geología de Chiapas. Gobierno del Estado de Chiapas. Tuxtla Gutiérrez, Chiapas, México.

National Geographic Society. 1999. Field guide to the birds of North America. $3^{\text {th }}$ edit. National Geographic Society. Washington, D.C.

Navarro S., A.G. \& H. Benítez. 1993. Patrones de riqueza y endemismo de las aves. Ciencias, 7: 4553.

Navarro S., A.G. \& A.T. Peterson. 2004. An alternative species taxonomy of the birds of Mexico. Biota Neotropica, 1: 1-33.

Navarro S., A.G. \& L.A.G. Sánchez-González. 2003. La diversidad de las aves. Pp. 24-86. In: H. Gómez de Silva and A. Oliveras de Ita (Eds.). Conservación de aves. Experiencias en México. Cipamex-Conabio-National Fish and Wildlife Foundation. México, D.F.

Navarro S., A.G., A.T. Peterson \& A. Gordillo. 2003. Museums working together: the atlas of the birds of Mexico. In: N. Collar, C. Ficher and C. Feare (Eds.). Why museums matter: avian archives in an age of extinction. Bulletin British OrnithologiSt'Club Supplement, 123A: 207-225.

Ortiz-Pulido, R., H. Gómez de Silva, F. González-García \& A. Álvarez. 1995. Avifauna del Centro de Investigaciones Costeras La Mancha, Veracruz, México. Acta Zoológica Mexicana (n. s.), 66: 87-118. 
Ovando D., L. 1990. Avifauna del Parque Nacional Cañón del Sumidero, Chiapas, México. Tesis de Licenciatura. Instituto de Ciencias y Artes de Chiapas. Tuxtla Gutiérrez, Chiapas, México.

Palomera-García, C., E. Santana \& R. Amparán-Salcido. 1994. Patrones de distribución de la avifauna en tres estados del occidente de México. Anales del Instituto de Biología, UNAM, Serie Zoología, 5: 137-175.

Peterson, R.T. \& E.L. Chalif. 1989. Aves de México: Guía de campo. Ed. Diana. México, D.F.

Pettingill, O.S.Jr. 1969. Ornithology in laboratory and field. 4th ed. Burgués, Minneapolis, MN.

Ralph, C.J., J.R. Saber \& S. Droege. 1995. Monitoring bird populations by point counts. General Technical Report PSW-GTR-149. USDA Forest Service, Pacific Southwest Research Station. Albany, CA.

Ramírez-Albores, J.E. 2004. Efecto de la estructura del paisaje sobre la diversidad $\alpha$, $\beta$ y $\gamma$ de comunidades de aves en San Fernando, Chiapas, México. Tesis de Maestría, El Colegio de la Frontera Sur. San Cristóbal de las Casas, Chiapas, México.

Ramírez-Albores, J.E. 2010. Diversidad de aves de hábitats naturales y modificados en un paisaje de la Depresión Central de Chiapas, México. Revista de Biología Tropical, 58: 511-528.

Ramírez-Albores, J.E. \& G. Ramírez. 2002. Avifauna de la región oriente de la Sierra de Huautla, Morelos, México. Anales del Instituto de Biología, UNAM, Serie Zoología, 73: 91-111.

Rangel-Salazar, J., P. Enríquez \& T. Hill. 2005. Diversidad de aves en Chiapas: prioridades de investigación para su conservación. Pp. 265-323. In: M. González-Espinosa, N. Ramírez-Marcial and L. Ruiz-Montoya (Eds.). La diversidad biológica en Chiapas. Ed. Plaza y Valdés. México.

Rangel-Salazar, J., P. Enríquez \& E. Sántiz. 2009. Variación de la diversidad de aves de sotobosque en el Parque Nacional Lagos de Montebello, Chiapas, México. Acta Zoologica Mexicana (n.s.), 25: 479-495.

Reyes, A. \& M. Souza. 1997. Listados florísticos de México XVII: Depresión Central de Chiapas. La selva baja caducifolia. Instituto de Biología, UNAM. México, D.F.

Rickelfs, R.E. \& D. Schluter. 1993. Species diversity: regional and historical influences. Pp 350-363. In: R.E. Rickelfs and D. Schluter (Eds). Species diversity in Ecological Communities. Historical and Geographical Perspectives. University of Chicago Press, Chicago.

Ríos M., C. 2006. Patrones biogeográficos de la avifauna de las selvas secas de Mesoamérica. Tesis de Maestría. Facultad de Ciencias, UNAM. México, D.F.

Rojas-Soto, O. \& A. Oliveras de Ita. 2005. Los inventarios avifaunísticos: reflexiones sobre su desarrollo en el neotrópico. Ornitología Neotropical, 16:441-445.

Trejo, I. 1998. Distribución y diversidad de selvas bajas en México: relaciones con el clima y el suelo. Tesis de Doctorado. Facultad de Ciencias, UNAM. México, D.F.

Whittaker, R.J., K.J. Willis \& R. Field. 2001. Scale and species richness: towards a general hierarchical theory of species diversity. Journal of Biogeography, 28: 453-470. 


\section{APÉNDICE 1}

Listado de la avifauna registrada en 24 sitios de muestreo ubicados en la Depresión Central de Chiapas de 2003 al 2004. El orden taxonómico se basa en AOU (2008). Estatus migratorio: residente $(\mathrm{R})$, visitante de invierno (W), residente de verano (S), transitoria (T). Abundancia relativa: rara (R), no común $(\mathrm{I})$, moderadamente común $(\mathrm{E})$, común $(\mathrm{C})$, abundante $(\mathrm{A})$. Tipo de vegetación: $\mathrm{SB}$ (selva baja caducifolia), Pp (pastizal/potrero), Cv (cercos vivos), Vr (vegetación riparia), ST (selva mediana subperennifolia), Vs (vegetación secundaria), C (cultivo), Et (encinar tropical), Us (zona urbana y suburbana, incluye parques y centros de recreación) y Ac (acuáticas y subacuáticas). Estatus de conservación: NOM-059-SEMARNAT-2001 (Pr, bajo protección especial; A, amenazada; P, en

peligro de extinción), IUCN (NT, casi amenazada; VU, vulnerable). ${ }^{1}$ Especie endémica a Mesoamérica; 2 Especie endémica al oeste de México; ${ }^{3}$ Especie asociada a la selva baja caducifolia. -Registros obtenidos de colecciones y/o literatura para los sitios de estudio, cuya presencia no fue corroborada en este estudio. $\square$ Registros obtenidos de colecciones y/o literatura para los sitios de estudio, cuya presencia fue corroborada en este estudio. $\nabla$ Registros provenientes de observaciones en campo durante la realización del presente estudio. ${ }^{\circ}$ Registros provenientes de observaciones realizadas en campo y de colecciones y/o literatura, que de acuerdo a Howell y Webb (1995) se distribuyen en esta región pero no son considerados por Rangel-Salazar et al. (2005). Registros adicionales realizados en el presente estudio para la región de la Depresión Central de Chiapas ( $)$ y para el Parque Nacional Cañón del Sumidero (•).

\begin{tabular}{|c|c|c|c|c|}
\hline & $\begin{array}{c}\text { Estatus } \\
\text { migratorio }\end{array}$ & $\begin{array}{c}\text { Abundancia } \\
\text { relativa }\end{array}$ & $\begin{array}{c}\text { Tipos de } \\
\text { vegetación }\end{array}$ & $\begin{array}{c}\text { Estatus de } \\
\text { conservación }\end{array}$ \\
\hline Crypturellus soui $\mathbf{~} \bullet$ & $\mathrm{R}$ & E & SB & $\operatorname{Pr}$ \\
\hline Crypturellus cinnamomeus $\square \mathbf{1}^{1,3^{\circ}} \uparrow$ & $\mathrm{R}$ & I & $\mathrm{SB}, \mathrm{ST}$ & \\
\hline Dendrocygna autumnalis $\square \uparrow$ & $\mathrm{R}$ & I & Ac & \\
\hline Anas discors $\square$ & $\mathrm{W}$ & I & Ac & \\
\hline Ortalis vetula & $\mathrm{R}$ & $\mathrm{C}$ & $\mathrm{SB}, \mathrm{ST}, \mathrm{Vs}, \mathrm{Et}$ & \\
\hline Penelope purpurascens $\square^{3} 3^{\circ}$ & $\mathrm{R}$ & $\mathrm{R}$ & $\mathrm{SB}, \mathrm{ST}$ & $\mathrm{A}$, \\
\hline Penelopina nigra $\bullet \mathbf{\bullet}, \mathbf{3}_{\uparrow}$ & $\mathrm{R}$ & & & $\mathrm{A}, \mathrm{VU}$ \\
\hline Crax rubra $\bullet \mathbf{3}_{\uparrow}$ & $\mathrm{R}$ & & & $\mathrm{A}, \mathrm{VU}$ \\
\hline Colinus virginianus $\square$ & $\mathrm{R}$ & $\mathrm{C}$ & Vs, Pp, C & NT \\
\hline Dactylortyx thoracicus $\bullet \mathbf{1}, \mathbf{3}_{\uparrow}$ & $\mathrm{R}$ & & & $\operatorname{Pr}$ \\
\hline Cyrtonyx ocellatus $\bullet \mathbf{1}_{\uparrow}$ & $\mathrm{R}$ & & & A, NT \\
\hline Tachybaptus dominicus ם & $\mathrm{R}$ & I & $\mathrm{Ac}$ & $\operatorname{Pr}$ \\
\hline Podilymbus podiceps $\mathbf{v}$ & $\mathrm{W}$ & I & Ac & \\
\hline Pelecanus erythrorhynchos $\bullet$ & $\mathrm{W}$ & & Ac & \\
\hline Pelecanus occidentalis $\square$ \ & $\mathrm{O}$ & $\mathrm{R}$ & Ac & \\
\hline Phalacrocorax brasilianus & $\mathrm{R}$ & E & Ac & \\
\hline Anhinga anhinga $\boldsymbol{v}$ & $\mathrm{R}$ & I & Ac & \\
\hline Ixobrychus exilis • & W & & $\mathrm{Ac}$ & \\
\hline Ardea herodias $\square \mathbf{v}$ & W & $\mathrm{R}$ & $\mathrm{Ac}$ & \\
\hline
\end{tabular}




\begin{tabular}{|c|c|c|c|c|}
\hline & $\begin{array}{c}\text { Estatus } \\
\text { migratorio }\end{array}$ & $\begin{array}{c}\text { Abundancia } \\
\text { relativa }\end{array}$ & $\begin{array}{c}\text { Tipos de } \\
\text { vegetación }\end{array}$ & $\begin{array}{l}\text { Estatus de } \\
\text { conservación }\end{array}$ \\
\hline Ardea alba $\square^{\circ} \uparrow \uparrow$ & W & $\mathrm{R}$ & Ac & \\
\hline Egretta thula $\square^{\circ} \uparrow \uparrow$ & $\mathrm{W}$ & I & Ac & \\
\hline Egretta caerulea $\mathbf{\nabla}$ & W & $\mathrm{R}$ & Ac & \\
\hline Egretta tricolor $\bullet^{\circ}{ }_{\uparrow}$ & $\mathrm{W}$ & & Ac & \\
\hline Bubulcus ibis $\square^{\circ} \vee \uparrow$ & $\mathrm{R}$ & $\mathrm{C}$ & $\mathrm{C}$ & \\
\hline Butorides virescens ${\square^{\circ}}^{\circ} \uparrow$ & $\mathrm{W}$ & $\mathrm{R}$ & Ac & \\
\hline Nycticorax nycticorax $\square$ & $\mathrm{W}$ & $\mathrm{R}$ & Ac & \\
\hline Nyctinassa violacea $\square^{\circ}{ }^{\circ}$ & $\mathrm{R}$ & I & Ac & \\
\hline Coragyps atratus ם & $\mathrm{R}$ & A & $\mathrm{SB}, \mathrm{ST}, \mathrm{Vs}, \mathrm{Et}, \mathrm{Pp}, \mathrm{C}, \mathrm{Vr}, \mathrm{U}$ & \\
\hline Cathartes aura $\square \mathbf{v}$ & $\mathrm{R}$ & A & $\mathrm{SB}, \mathrm{ST}, \mathrm{Vs}, \mathrm{Et}, \mathrm{Pp}, \mathrm{C}, \mathrm{Vr}, \mathrm{U}$ & \\
\hline Pandion haliaetus $\square^{\circ} \uparrow$ & $\mathrm{T}$ & $\mathrm{R}$ & $\mathrm{SB}, \mathrm{ST}, \mathrm{Vs}$ & \\
\hline Chondrohierax uncinatus $\boldsymbol{\bullet}^{3^{\circ}}{ }$ & $\mathrm{R}$ & & & $\operatorname{Pr}$ \\
\hline Elanus leucurus $\mathbf{\nabla}$ & $\mathrm{R}$ & $\mathrm{R}$ & $\mathrm{Pp}, \mathrm{C}$ & \\
\hline Rostrhamus sociabilis & $\mathrm{R}$ & $\mathrm{R}$ & ST, Vs & $\operatorname{Pr}$ \\
\hline Ictinia mississippiensis $\boldsymbol{}^{\circ} \downarrow$ & $\mathrm{T}$ & I & $\mathrm{SB}, \mathrm{ST}, \mathrm{Et}, \mathrm{Pp}, \mathrm{C}$ & $\operatorname{Pr}$ \\
\hline Accipiter striatus $\square \mathbf{v}$ & $\mathrm{W}$ & $\mathrm{R}$ & $\mathrm{SB}, \mathrm{ST}, \mathrm{Vs}, \mathrm{Pp}, \mathrm{C}, \mathrm{Us}$ & $\operatorname{Pr}$ \\
\hline Accipiter cooperii $\square$ & $\mathrm{W}$ & $\mathrm{E}$ & $\mathrm{SB}, \mathrm{ST}, \mathrm{Vs}, \mathrm{Et}, \mathrm{Pp}, \mathrm{C}$ & $\operatorname{Pr}$ \\
\hline Buteogallus anthracinus $\square \mathbf{3} \mathbf{\imath}$ & $\mathrm{R}$ & $\mathrm{E}$ & $\mathrm{SB}, \mathrm{Vs}, \mathrm{Pp}, \mathrm{C}$ & $\operatorname{Pr}$ \\
\hline Buteo magnirostris $\square^{3}{ }^{\circ} \uparrow$ & $\mathrm{R}$ & $\mathrm{E}$ & $\mathrm{SB}, \mathrm{Vs}, \mathrm{Et}, \mathrm{Pp}, \mathrm{C}$ & \\
\hline Buteo platypterus $\bullet$ & $\mathrm{T}$ & & & $\operatorname{Pr}$ \\
\hline Buteo nitidus ם & $\mathrm{R}$ & I & $\mathrm{SB}, \mathrm{Vs}, \mathrm{Pp}, \mathrm{C}$ & \\
\hline Buteo brachyurus $\square \mathbf{3} \mathbf{v}$ & $\mathrm{R}$ & I & $\mathrm{SB}, \mathrm{Vs}$ & \\
\hline Buteo swainsoni ष & $\mathrm{T}$ & $\mathrm{R}$ & $\mathrm{SB}, \mathrm{Vs}, \mathrm{Pp}$ & $\operatorname{Pr}$ \\
\hline Buteo albicaudatus ם & $\mathrm{R}$ & I & $\mathrm{SB}, \mathrm{ST}$ & $\operatorname{Pr}$ \\
\hline Buteo albonotatus • & $\mathrm{W}$ & & $\mathrm{SB}, \mathrm{ST}, \mathrm{Vs}, \mathrm{Pp}$ & \\
\hline Buteo jamaicensis ם & $\mathrm{R}$ & $\mathrm{E}$ & $\mathrm{SB}, \mathrm{Vs}, \mathrm{Pp}, \mathrm{C}, \mathrm{Us}$ & \\
\hline Caracara cheriway $\mathbf{v}$ & $\mathrm{R}$ & $\mathrm{R}$ & $\mathrm{Pp}, \mathrm{C}$ & \\
\hline Herpetotheres cachinnans $3^{3^{\circ} \uparrow}$ & $\mathrm{R}$ & I & $\mathrm{Pp}, \mathrm{C}$ & \\
\hline Falco sparverius ם & $\mathrm{R}$ & $\mathrm{E}$ & $\mathrm{Pp}, \mathrm{C}$ & \\
\hline Falco columbarius $\square$ & W & $\mathrm{R}$ & Vs, Pp,C & \\
\hline Falco femoralis $\mathbf{v}$ & $\mathrm{R}$ & $\mathrm{R}$ & $\mathrm{Pp}, \mathrm{C}$ & A \\
\hline Falco rufigularis $\cdot \mathbf{3}^{\circ}{ }_{\uparrow}$ & $\mathrm{R}$ & & & \\
\hline Falco peregrinus $\square$ & W & I & $\mathrm{Pp}, \mathrm{C}$ & $\operatorname{Pr}$ \\
\hline Aramides cajanea $\bullet \bullet$ & $\mathrm{R}$ & & $\mathrm{Ac}$ & \\
\hline Porzana carolina $\bullet \bullet$ & W & & & \\
\hline Fulica americana $\bullet$ & $\mathrm{R}$ & & & \\
\hline
\end{tabular}




\begin{tabular}{|c|c|c|c|c|}
\hline & $\begin{array}{c}\text { Estatus } \\
\text { migratorio }\end{array}$ & $\begin{array}{c}\text { Abundancia } \\
\text { relativa }\end{array}$ & $\begin{array}{c}\text { Tipos de } \\
\text { vegetación }\end{array}$ & $\begin{array}{c}\text { Estatus de } \\
\text { conservación }\end{array}$ \\
\hline Charadrius collaris $\bullet \bullet$ & $\mathrm{R}$ & & & \\
\hline Charadrius vociferus $\mathbf{~} \bullet$ & W & I & Ac & \\
\hline Himantopus mexicanus $\mathbf{v}$ & $\mathrm{R}$ & $\mathrm{E}$ & Ac & \\
\hline Jacana spinosa $\square$ & $\mathrm{R}$ & I & Ac & \\
\hline Actitis macularius $\mathbf{} \square$ & W & $\mathrm{R}$ & Ac & \\
\hline Tringa solitaria $\mathbf{v}$ & W & $\mathrm{R}$ & Ac & \\
\hline Calidris melanotus $\bullet$ & $\mathrm{T}$ & & & \\
\hline Calidris minutilla $\bullet$ & W & & & \\
\hline Gallinago gallinago $\bullet$ & W & & & \\
\hline Leucophaeus pipixcan • & $\mathrm{T}$ & & & \\
\hline Columba livia $\mathbf{v}$ & $\mathrm{R}$ & $\mathrm{C}$ & Us & \\
\hline Patagioenas flavirostris $\square \mathbf{1}, \mathbf{3} \mathbf{v}$ & $\mathrm{R}$ & $\mathrm{E}$ & $\mathrm{SB}, \mathrm{ST}, \mathrm{Vs}$ & \\
\hline Patagioenas nigrirostris $\mathbf{1} \mathbf{\uparrow}$ & $\mathrm{R}$ & I & $\mathrm{SB}, \mathrm{Vs}, \mathrm{C}$ & \\
\hline Zenaida asiatica $\square \mathbf{v}$ & $\mathrm{R}$ & A & $\mathrm{SB}, \mathrm{ST}, \mathrm{Vs}, \mathrm{C}, \mathrm{Us}$ & \\
\hline Zenaida macroura $\square \mathbf{v}$ & W & I & $\mathrm{SB}, \mathrm{ST}, \mathrm{Vs}, \mathrm{C}$ & \\
\hline Columbina inca $\square \mathbf{v}$ & $\mathrm{R}$ & A & Vs, Et, Pp, C, Us & \\
\hline Columbina passerina $\square \mathbf{v}$ & $\mathrm{R}$ & $\mathrm{E}$ & Vs, Pp,C & \\
\hline Columbina minuta ${ }^{\circ} \uparrow$ & $\mathrm{R}$ & $\mathrm{E}$ & $\mathrm{SB}, \mathrm{Vs}, \mathrm{Vr}$ & \\
\hline Columbina talpacoti $\square \mathbf{v}$ & $\mathrm{R}$ & $\mathrm{R}$ & Vs, Pp,C & \\
\hline Claravis petriosa & $\mathrm{R}$ & $\mathrm{R}$ & $\mathrm{SB}, \mathrm{Vs}$ & \\
\hline Claravis mondeteura $\bullet \uparrow$ & $\mathrm{R}$ & & & \\
\hline Leptotila verreauxi $\square \mathbf{} \mathbf{3}$ & $\mathrm{R}$ & $\mathrm{C}$ & $\mathrm{SB}, \mathrm{ST}, \mathrm{Vs}, \mathrm{C}, \mathrm{Vr}$ & \\
\hline Leptotila plumbeiceps $\bullet \uparrow$ & $\mathrm{R}$ & & & \\
\hline Geotrygon montana $\square^{3}{ }^{\circ} \uparrow$ & $\mathrm{R}$ & $\mathrm{R}$ & $\mathrm{SB}, \mathrm{Vs}$ & \\
\hline Aratinga holochlora $\square \mathbf{1 v}$ & $\mathrm{R}$ & $\mathrm{R}$ & $\mathrm{SB}, \mathrm{ST}$ & $\operatorname{Pr}$ \\
\hline Aratinga nana $\square \mathbf{1} \mathbf{v} \bullet$ & $\mathrm{R}$ & $\mathrm{C}$ & SB, Vs, Us & $\operatorname{Pr}$ \\
\hline Aratinga canicularis $\square \mathbf{1}, \mathbf{3} \mathbf{\uparrow}$ & $\mathrm{R}$ & $\mathrm{E}$ & $\mathrm{SB}, \mathrm{ST}, \mathrm{Vs}, \mathrm{C}, \mathrm{Us}$ & $\operatorname{Pr}$ \\
\hline Amazona albifrons $\square \mathbf{\square}, \mathbf{3} \mathbf{\imath}$ & $\mathrm{R}$ & $\mathrm{E}$ & $\mathrm{SB}, \mathrm{ST}$ & \\
\hline Amazona autumnalis $3 \mathbf{v}$ & $\mathrm{R}$ & I & SB, ST, Us & \\
\hline Coccyzus americanus $\bullet$ & $\mathrm{T}$ & & $\mathrm{SB}, \mathrm{Vs}$ & \\
\hline Coccyzus minor $\square^{3} \mathbf{v}$ & $\mathrm{R}$ & $\mathrm{E}$ & $\mathrm{SB}, \mathrm{ST}, \mathrm{Vs}$ & \\
\hline Piaya cayana $\mathbf{\square} \mathbf{1 , 3} \mathbf{v}$ & $\mathrm{R}$ & $\mathrm{C}$ & $\mathrm{SB}, \mathrm{ST}, \mathrm{Vs}$ & \\
\hline Tapera naevia $\square \mathbf{v}$ & $\mathrm{R}$ & $\mathrm{E}$ & $\mathrm{SB}, \mathrm{C}$ & \\
\hline Dromococcyx phasianellus $\mathbf{\square} \mathbf{3}$ & $\mathrm{R}$ & I & $\mathrm{SB}, \mathrm{ST}$ & \\
\hline Morococcyx erythropygus 미 & $\mathrm{R}$ & $\mathrm{E}$ & $\mathrm{SB}, \mathrm{ST}, \mathrm{Vs}$ & \\
\hline Geococcyx velox ㅁ1 & $\mathrm{R}$ & $\mathrm{E}$ & $\mathrm{Vs}, \mathrm{Pp}, \mathrm{C}$ & \\
\hline
\end{tabular}




\begin{tabular}{|c|c|c|c|c|}
\hline & $\begin{array}{c}\text { Estatus } \\
\text { migratorio }\end{array}$ & $\begin{array}{c}\text { Abundancia } \\
\text { relativa }\end{array}$ & $\begin{array}{c}\text { Tipos de } \\
\text { vegetación }\end{array}$ & $\begin{array}{c}\text { Estatus de } \\
\text { conservación }\end{array}$ \\
\hline Crotophaga sulcirostris $\square$ & $\mathrm{R}$ & $\mathrm{C}$ & Vs, Pp,C, Cv, Us & \\
\hline Tyto alba & $\mathrm{R}$ & $\mathrm{R}$ & $\mathrm{SB}, \mathrm{Pp}, \mathrm{C}$ & \\
\hline Megascops trichopsis $\bullet 1$ & $\mathrm{R}$ & & & \\
\hline Megascops barbarus $\boldsymbol{\bullet}_{\uparrow}$ & $\mathrm{R}$ & & & A, NT \\
\hline Megascops guatemalae $\square \mathbf{3} \mathbf{v} \uparrow$ & $\mathrm{R}$ & I & $\mathrm{SB}, \mathrm{ST}, \mathrm{Vs}$ & \\
\hline Pulsatrix perspicillata $\mathbf{3} \mathbf{v}$ & $\mathrm{R}$ & $\mathrm{R}$ & $\mathrm{SB}, \mathrm{ST}, \mathrm{Pp}, \mathrm{C}$ & A \\
\hline Glaucidium griseiceps $\bullet \uparrow$ & $\mathrm{R}$ & & & $\operatorname{Pr}$ \\
\hline Glaucidium brasilianum 口 & $\mathrm{R}$ & I & $\mathrm{SB}, \mathrm{ST}, \mathrm{Vs}$ & \\
\hline Ciccaba virgata $\square \mathbf{3} \mathbf{v}$ & $\mathrm{R}$ & $\mathrm{R}$ & $\mathrm{SB}, \mathrm{ST}$ & \\
\hline Chordeiles acutipennis ם & $\mathrm{R}$ & $\mathrm{E}$ & SB, ST, Vs, Us & \\
\hline Chordeiles minor $\bullet$ & $\mathrm{S}$ & & SB & \\
\hline Nyctidromus albicollis $\square \mathbf{3} \mathbf{v}$ & $\mathrm{R}$ & $\mathrm{R}$ & SB, ST, Vs, Us & \\
\hline Caprimulgus carolinensis $\bullet$ & $\mathrm{W}$ & & & \\
\hline Caprimulgus ridgwayi $\square \mathbf{1 , 3 \mathbf { }}$ & $\mathrm{R}$ & $\mathrm{E}$ & $\mathrm{SB}, \mathrm{ST}, \mathrm{Vs}$ & \\
\hline Caprimulgus vociferus • & W & & $\mathrm{SB}, \mathrm{ST}, \mathrm{Vs}$ & \\
\hline Nyctibius jamaicensis • & $\mathrm{R}$ & & & \\
\hline Streptoprocne zonaris ם & $\mathrm{R}$ & I & $\mathrm{SB}, \mathrm{ST}, \mathrm{Vs}, \mathrm{Et}, \mathrm{Pp}, \mathrm{C}$ & \\
\hline Chaetura vauxi $\square \mathbf{v}$ & $\mathrm{R}$ & $\mathrm{E}$ & $\mathrm{SB}, \mathrm{ST}, \mathrm{Vs}, \mathrm{Et}, \mathrm{Pp}, \mathrm{C}, \mathrm{Us}$ & \\
\hline Aeronautes saxatalis $\square \mathbf{v}$ & $\mathrm{R}$ & $\mathrm{E}$ & $\mathrm{SB}, \mathrm{ST}, \mathrm{Vs}, \mathrm{Et}, \mathrm{Pp}, \mathrm{C}$ & \\
\hline Panyptila sanctihieronymi $\square \mathbf{\square} \mathbf{v}$ & $\mathrm{R}$ & I & $\mathrm{SB}, \mathrm{ST}, \mathrm{Vs}, \mathrm{Et}, \mathrm{Pp}, \mathrm{C}$ & \\
\hline Phaethornis longirostris $\mathbf{1 , 3} \mathbf{v}^{\circ}$ & $\mathrm{R}$ & $\mathrm{E}$ & $\mathrm{SB}, \mathrm{Vs}$ & \\
\hline Phaethornis striigularis $\boldsymbol{\vee}^{\circ} \bullet$ & $\mathrm{R}$ & I & SB & $\operatorname{Pr}$ \\
\hline Campylopterus hemileucurus $\boldsymbol{\bullet}_{\uparrow}$ & $\mathrm{R}$ & & & \\
\hline Florigusa mellivora $\mathbf{3} \mathbf{v} \bullet$ & $\mathrm{R}$ & $\mathrm{R}$ & $\mathrm{SB}, \mathrm{ST}$ & \\
\hline Colibri thalassinus $\mathbf{v}$ & $\mathrm{R}$ & $\mathrm{E}$ & SB, Vs, Us & \\
\hline Abeillia abeillei $\bullet \uparrow$ & $\mathrm{R}$ & & & $\operatorname{Pr}$ \\
\hline Chlorostilbon canivetii $\square \mathbf{1}, \mathbf{3} \mathbf{v}$ & $\mathrm{R}$ & $\mathrm{C}$ & SB, ST, Vs, Us & \\
\hline Hylocharis leucotis $\bullet \mathbf{1}$ & $\mathrm{R}$ & & & \\
\hline Amazilia candida $\bullet \mathbf{1}, \mathbf{3}_{\uparrow}$ & $\mathrm{R}$ & & & \\
\hline Amazilia cyanocephala $\bullet^{\mathbf{o}_{\uparrow}}$ & $\mathrm{R}$ & & & \\
\hline Amazilia beryllina $\square \mathbf{1 , 3 \mathbf { v }}$ & $\mathrm{R}$ & I & $\mathrm{SB}, \mathrm{ST}, \mathrm{Cv}, \mathrm{Vr}$ & \\
\hline Amazilia tzacatl $\square 3 \mathbf{v \uparrow}$ & $\mathrm{R}$ & $\mathrm{C}$ & $\mathrm{SB}, \mathrm{Vs}, \mathrm{Cv}, \mathrm{Vr}, \mathrm{Us}$ & \\
\hline Amazilia yucatanensis $\square \mathbf{1 , 3} \mathbf{\mathbf { v } \uparrow}$ & $\mathrm{R}$ & $\mathrm{E}$ & $\mathrm{SB}, \mathrm{Vs}$ & \\
\hline Amazilia viridifrons $\square \mathbf{1 , 2 , 3} \mathbf{v}$ & $\mathrm{R}$ & $\mathrm{C}$ & $\mathrm{SB}, \mathrm{ST}, \mathrm{Vs}$ & A \\
\hline Eupherusa eximia $\square \mathbf{1} \mathbf{\nabla} \uparrow$ & $\mathrm{R}$ & I & $\mathrm{SB}, \mathrm{ST}, \mathrm{Vs}$ & \\
\hline Lampornis viridipallens $\bullet \mathbf{1}$ & $\mathrm{R}$ & & & $\operatorname{Pr}$ \\
\hline
\end{tabular}




\begin{tabular}{|c|c|c|c|c|}
\hline & $\begin{array}{c}\text { Estatus } \\
\text { migratorio }\end{array}$ & $\begin{array}{c}\text { Abundancia } \\
\text { relativa }\end{array}$ & $\begin{array}{c}\text { Tipos de } \\
\text { vegetación }\end{array}$ & $\begin{array}{c}\text { Estatus de } \\
\text { conservación }\end{array}$ \\
\hline Lamprolaima rhami $\mathbf{1} \mathbf{v}$ & $\mathrm{R}$ & $\mathrm{R}$ & $\mathrm{SB}, \mathrm{ST}$ & $\mathrm{A}$ \\
\hline Eugenes fulgens • & $\mathrm{R}$ & & & \\
\hline Heliomaster longirostris $\square \mathbf{3}$ & $\mathrm{R}$ & $\mathrm{R}$ & $\mathrm{SB}, \mathrm{Vs}$ & $\operatorname{Pr}$ \\
\hline Heliomaster constantii $\bullet \mathbf{1 , 3}$ & $\mathrm{R}$ & & & \\
\hline Doricha enicura $\bullet 1$ & $\mathrm{R}$ & & & $\operatorname{Pr}$ \\
\hline Tilmatura dupontii $\square \mathbf{1} \mathbf{v}$ & $\mathrm{R}$ & I & $\mathrm{SB}, \mathrm{ST}, \mathrm{Vs}$ & A \\
\hline Archilochus colubris ם & W & $\mathrm{R}$ & SB & \\
\hline Trogon melanocephalus $\mathbf{1 , 3 v}$ & $\mathrm{R}$ & $\mathrm{C}$ & $\mathrm{SB}, \mathrm{ST}$ & \\
\hline Trogon violaceus $\square^{3} 3^{\circ} \uparrow$ & $\mathrm{R}$ & $\mathrm{R}$ & $\mathrm{SB}, \mathrm{ST}$ & \\
\hline Trogon collaris $\bullet^{\circ} \uparrow$ & $\mathrm{R}$ & & & $\operatorname{Pr}$ \\
\hline Momotus mexicanus $\square \mathbf{1 , 3 \mathbf { v }}$ & $\mathrm{R}$ & $\mathrm{C}$ & $\mathrm{SB}, \mathrm{ST}, \mathrm{Et}, \mathrm{Vr}$ & \\
\hline Momotus momota $\square \square^{1,3^{\circ}}{ }^{\uparrow}$ & $\mathrm{R}$ & $\mathrm{E}$ & $\mathrm{SB}, \mathrm{ST}, \mathrm{Vr}$ & \\
\hline Megaceryle torquata $\square \mathbf{v}$ & $\mathrm{R}$ & I & $\mathrm{Vr}, \mathrm{Ac}$ & \\
\hline Megaceryle alcyon $\square \mathbf{v}$ & W & $\mathrm{R}$ & $\mathrm{Vr}, \mathrm{Ac}$ & \\
\hline Chloroceryle amazona $\square \mathbf{v}$ & $\mathrm{R}$ & $\mathrm{R}$ & Vr, Ac & \\
\hline Chloroceryle americana $\square \mathbf{v}$ & $\mathrm{R}$ & I & $\mathrm{Vr}, \mathrm{Ac}$ & \\
\hline Aulacorhynchus prasinus $\square$ & $\mathrm{R}$ & $\mathrm{R}$ & ST, Vs & $\operatorname{Pr}$ \\
\hline Pteroglossus torquatus $\square \mathbf{\uparrow}$ & $\mathrm{R}$ & $\mathrm{R}$ & ST, Vs & \\
\hline Ramphastos sulfuratus & $\mathrm{R}$ & $\mathrm{R}$ & ST, Vs & A \\
\hline Melanerpes formicivorus • & $\mathrm{R}$ & & & \\
\hline Melanerpes aurifrons $\square \mathbf{3} \mathbf{v}$ & $\mathrm{R}$ & $\mathrm{C}$ & SB, ST, Vs, Et, Us & \\
\hline Sphyrapicus varius & W & $\mathrm{R}$ & $\mathrm{SB}, \mathrm{ST}, \mathrm{Vs}$ & \\
\hline Picoides scalaris $\square \mathbf{\square} \mathbf{v}$ & $\mathrm{R}$ & $\mathrm{R}$ & $\mathrm{SB}, \mathrm{Vs}, \mathrm{Us}$ & \\
\hline Veniliornis fumigatus $\bullet \uparrow$ & $\mathrm{R}$ & & & \\
\hline Piculus rubiginosus $\square \mathbf{1 , 3} \mathbf{v}$ & $\mathrm{R}$ & I & $\mathrm{SB}, \mathrm{ST}, \mathrm{Vs}$ & \\
\hline Dryocopus lineatus $\square \mathbf{3} \mathbf{v}$ & $\mathrm{R}$ & $\mathrm{R}$ & $\mathrm{SB}, \mathrm{ST}, \mathrm{Vs}$ & \\
\hline Campephilus guatemalensis $\mathbf{1 , 3 ^ { \circ }}{ }^{\star}$ & $\mathrm{R}$ & I & $\mathrm{SB}, \mathrm{ST}, \mathrm{Vs}$ & $\operatorname{Pr}$ \\
\hline Sclerurus guatemalensis $\mathbf{v}$ & $\mathrm{R}$ & $\mathrm{R}$ & SB & $\operatorname{Pr}$ \\
\hline Synallaxis erythrothorax $1 \mathbf{v}$ & $\mathrm{R}$ & I & SB & \\
\hline Automolus ochrolaemus $\mathbf{\nabla}$ & $\mathrm{R}$ & $\mathrm{R}$ & $\mathrm{SB}, \mathrm{Vs}$ & $\operatorname{Pr}$ \\
\hline Dendrocincla anabatina $\bullet \uparrow$ & $\mathrm{R}$ & & & $\operatorname{Pr}$ \\
\hline Dendrocincla homochroa $3^{3 \circ} \uparrow$ & $\mathrm{R}$ & $\mathrm{R}$ & SB & \\
\hline Sittasomus griseicapillus $\mathbf{\bullet}^{\mathrm{O}_{\uparrow}}$ & $\mathrm{R}$ & & & \\
\hline Dendrocolaptes sanctithomae $\mathbf{3} \mathbf{v} \uparrow$ & $\mathrm{R}$ & $\mathrm{R}$ & SB & $\operatorname{Pr}$ \\
\hline Xiphorhynchus flavigaster $\square \mathbf{1 , 3}$ & $\mathrm{R}$ & $\mathrm{E}$ & $\mathrm{SB}, \mathrm{ST}$ & \\
\hline Lepidocolaptes souleyetii $\square \mathbf{3} \mathbf{v} \downarrow$ & $\mathrm{R}$ & $\mathrm{E}$ & $\mathrm{SB}, \mathrm{ST}, \mathrm{Vs}$ & \\
\hline
\end{tabular}




\begin{tabular}{|c|c|c|c|c|}
\hline & $\begin{array}{c}\text { Estatus } \\
\text { migratorio }\end{array}$ & $\begin{array}{c}\text { Abundancia } \\
\text { relativa }\end{array}$ & $\begin{array}{c}\text { Tipos de } \\
\text { vegetación }\end{array}$ & $\begin{array}{c}\text { Estatus de } \\
\text { conservación }\end{array}$ \\
\hline Lepidocolaptes affinis • & $\mathrm{R}$ & & & \\
\hline Taraba major $\square \uparrow$ & $\mathrm{R}$ & I & $\mathrm{SB}, \mathrm{Vs}, \mathrm{Cv}$ & $\operatorname{Pr}$ \\
\hline Thamnophilus doliatus $\square \mathbf{3} \mathbf{v}$ & $\mathrm{R}$ & $\mathrm{C}$ & $\mathrm{SB}, \mathrm{Vs}, \mathrm{C}, \mathrm{Cv}$ & \\
\hline Cercomacra tyrannina $\mathbf{\imath}$ & $\mathrm{R}$ & I & $\mathrm{SB}, \mathrm{Vs}$ & \\
\hline Grallaria guatemalensis $\square \mathbf{v}$ & $\mathrm{R}$ & $\mathrm{R}$ & $\mathrm{SB}, \mathrm{ST}$ & A \\
\hline Ornithion semiflavum $\mathbf{v} \bullet$ & $\mathrm{R}$ & $\mathrm{R}$ & SB & $\operatorname{Pr}$ \\
\hline Camptostoma imberbe $\mathbf{v}$ & $\mathrm{R}$ & I & $\mathrm{SB}, \mathrm{Vs}, \mathrm{Cv}, \mathrm{Us}$ & \\
\hline Myiopagis viridicata $\square^{3^{\circ}} \uparrow \uparrow$ & $\mathrm{R}$ & $\mathrm{E}$ & $\mathrm{SB}, \mathrm{Vs}$ & \\
\hline Elaenia flavogaster $\mathbf{v}$ & $\mathrm{R}$ & $\mathrm{R}$ & $\mathrm{SB}, \mathrm{Vs}$ & \\
\hline Mionectes oleagineus $\bullet \uparrow$ & $\mathrm{R}$ & & & \\
\hline Leptopogon amaurocephalus $\mathbf{v}$ & $\mathrm{R}$ & I & $\mathrm{SB}, \mathrm{Vs}$ & \\
\hline Oncostoma cinereigulare $\square \mathbf{1 , 3 \mathbf { v }}$ & $\mathrm{R}$ & $\mathrm{R}$ & $\mathrm{SB}, \mathrm{ST}, \mathrm{Vs}$ & \\
\hline Poecilotriccus sylvia & $\mathrm{R}$ & E & $\mathrm{SB}, \mathrm{ST}$ & \\
\hline Rhynchocyclus brevirostris $\square^{1}{ }^{\circ} \uparrow$ & $\mathrm{R}$ & I & $\mathrm{SB}, \mathrm{Vs}, \mathrm{Cv}$ & \\
\hline Tolmomyias sulphurescens $\bullet \mathbf{3}$ & $\mathrm{R}$ & & & \\
\hline Platyrinchus cancrominus $\bullet \mathbf{1}^{13^{\circ}} \uparrow$ & $\mathrm{R}$ & & & $\operatorname{Pr}$ \\
\hline Xenotriccus callizonus $\mathbf{1} \mathbf{1 , 3} \mathbf{v}$ & $\mathrm{R}$ & I & $\mathrm{SB}, \mathrm{Vs}$ & A, NT \\
\hline Contopus cooperi & W & $\mathrm{R}$ & $\mathrm{SB}, \mathrm{Vs}$ & \\
\hline Contopus pertinax $₫$ & $\mathrm{R}$ & $\mathrm{R}$ & $\mathrm{SB}, \mathrm{Vs}, \mathrm{Et}, \mathrm{Pp}, \mathrm{Cv}, \mathrm{Vr}$ & \\
\hline Contopus sordidulus • & $\mathrm{T}$ & & & \\
\hline Contopus virens & $\mathrm{T}$ & I & $\mathrm{SB}, \mathrm{Vs}$ & \\
\hline Contopus cinereus $₫ \uparrow$ & $\mathrm{R}$ & I & $\mathrm{SB}, \mathrm{Vs}, \mathrm{Et}, \mathrm{Pp}, \mathrm{Cv}, \mathrm{Vr}$ & \\
\hline Empidonax flaviventris $\bullet$ & W & & $\mathrm{SB}, \mathrm{Vs}$ & \\
\hline Empidonax virescens $\mathbf{v}$ & $\mathrm{T}$ & $\mathrm{R}$ & $\mathrm{SB}, \mathrm{Vs}$ & \\
\hline Empidonax traillii $\square$ & $\mathrm{T}$ & I & $\mathrm{SB}, \mathrm{ST}, \mathrm{Vs}$ & \\
\hline Empidonax albigularis $\square$ & $\mathrm{W}$ & $\mathrm{E}$ & $\mathrm{SB}, \mathrm{Vs}, \mathrm{Et}, \mathrm{Cv}, \mathrm{Vr}$ & \\
\hline Empidonax minimus 口 & $\mathrm{W}$ & $\mathrm{R}$ & $\mathrm{SB}, \mathrm{Vs}$ & \\
\hline Empidonax hammondii $\bullet$ & $\mathrm{T}$ & & & \\
\hline Empidonax flavescens $\bullet \mathbf{1}$ & $\mathrm{R}$ & & & \\
\hline Sayornis nigricans ㅁ & $\mathrm{R}$ & $\mathrm{R}$ & $\mathrm{SB}, \mathrm{Vs}, \mathrm{Vr}$ & \\
\hline Pyrocephalus rubinus ם & $\mathrm{R}$ & I & $\mathrm{Pp}, \mathrm{Cv}$ & \\
\hline Rhytipterna holerythra $\mathbf{\imath}$ & $\mathrm{R}$ & I & $\mathrm{SB}, \mathrm{Vs}$ & \\
\hline Myiarchus tuberculifer $\square$ & $\mathrm{R}$ & $\mathrm{R}$ & $\mathrm{SB}, \mathrm{Pp}, \mathrm{Cv}$ & \\
\hline Myiarchus cinerascens $\square$ & $\mathrm{W}$ & $\mathrm{R}$ & $\mathrm{SB}, \mathrm{ST}, \mathrm{Vs}$ & \\
\hline Myiarchus nuttingi $\square$ ロ1,3、 & $\mathrm{R}$ & $\mathrm{R}$ & $\mathrm{SB}, \mathrm{Vs}, \mathrm{Et}, \mathrm{Cv}, \mathrm{Vr}$ & \\
\hline Myiarchus crinitus $\bullet$ & $\mathrm{T}$ & & & \\
\hline
\end{tabular}




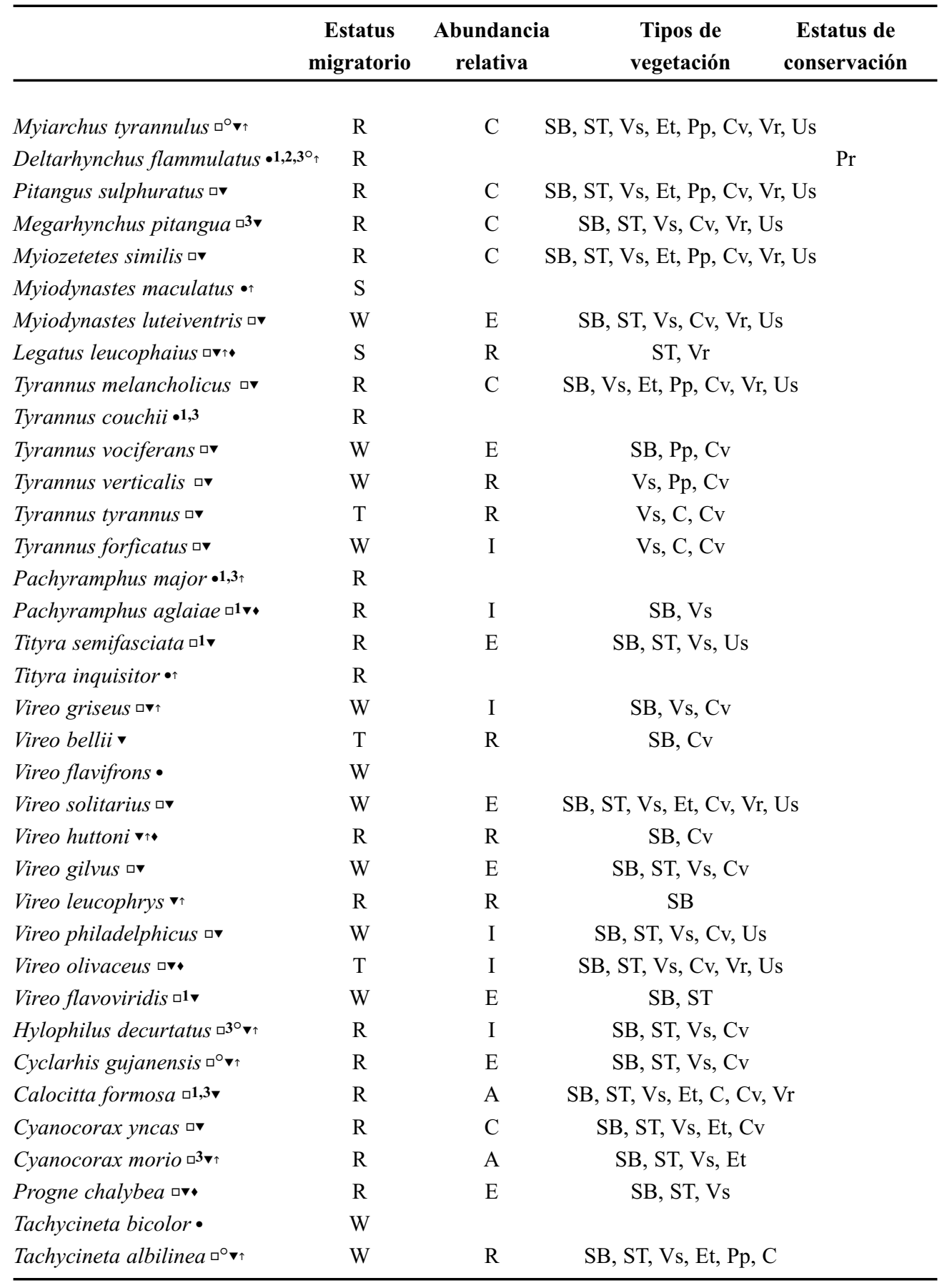




\begin{tabular}{|c|c|c|c|c|}
\hline & $\begin{array}{c}\text { Estatus } \\
\text { migratorio }\end{array}$ & $\begin{array}{c}\text { Abundancia } \\
\text { relativa }\end{array}$ & $\begin{array}{c}\text { Tipos de } \\
\text { vegetación }\end{array}$ & $\begin{array}{c}\text { Estatus de } \\
\text { conservación }\end{array}$ \\
\hline Stelgidopteryx serripennis $\square \mathbf{v}$ & $\mathrm{R}$ & $\mathrm{C}$ & SB, ST, Vs, Et, Pp, C, Us & \\
\hline Riparia riparia $\mathbf{~} \bullet$ & $\mathrm{T}$ & $\mathrm{E}$ & $\mathrm{ST}, \mathrm{Vr}$ & \\
\hline Petrochelidon pyrrhonota $\square \mathbf{v}$ & $\mathrm{T}$ & $\mathrm{R}$ & $\mathrm{Pp}, \mathrm{C}, \mathrm{Us}$ & \\
\hline Petrochelidon fulva $\square \mathbf{v}$ & S & $\mathrm{R}$ & $\mathrm{Pp}, \mathrm{C}, \mathrm{Us}$ & \\
\hline Hirundo rustica $\square \mathbf{v \bullet}$ & $\mathrm{W}$ & I & $\mathrm{Pp}, \mathrm{C}, \mathrm{Us}$ & \\
\hline Campylorhynchus zonatus $\bullet^{\circ_{\uparrow}}$ & $\mathrm{R}$ & & & \\
\hline Catherpes mexicanus $\mathbf{\square} \mathbf{v}$ & $\mathrm{R}$ & $\mathrm{E}$ & $\mathrm{SB}, \mathrm{ST}, \mathrm{Vr}$ & \\
\hline Thryothorus maculipectus $\mathbf{\square} \mathbf{1 , 3 \mathbf { v }}$ & $\mathrm{R}$ & $\mathrm{C}$ & $\mathrm{SB}, \mathrm{ST}, \mathrm{Vs}, \mathrm{Et}, \mathrm{Cv}$ & \\
\hline Thryothorus pleurostictus $\square \mathbf{1 , 3} \mathbf{}$ & $\mathrm{R}$ & $\mathrm{C}$ & $\mathrm{SB}, \mathrm{ST}, \mathrm{Vs}, \mathrm{Et}, \mathrm{C}, \mathrm{Cv}, \mathrm{Vr}$ & \\
\hline Thryothorus modestus $\square \mathbf{1 , 3} \mathbf{v}$ & $\mathrm{R}$ & $\mathrm{E}$ & $\mathrm{SB}, \mathrm{ST}, \mathrm{Vs}, \mathrm{C}, \mathrm{Cv}, \mathrm{Us}$ & \\
\hline Troglodytes aedon $\square^{\circ} \vee \uparrow$ & $\mathrm{R}$ & I & SB & \\
\hline Cistothorus platensis & $\mathrm{R}$ & $\mathrm{R}$ & SB & \\
\hline Uropsila leucogastra $\square \mathbf{1 , 3 \mathbf { v } \uparrow}$ & $\mathrm{R}$ & I & $\mathrm{SB}, \mathrm{ST}$ & \\
\hline Henicorchia leucosticta $\bullet \uparrow$ & $\mathrm{R}$ & & & \\
\hline Henicorchia leucophrys $\square^{\circ} \uparrow$ & $\mathrm{R}$ & I & $\mathrm{SB}, \mathrm{Vs}$ & \\
\hline Microcerculus marginatus $\mathbf{v}$ & $\mathrm{R}$ & $\mathrm{R}$ & ST & \\
\hline Ramphocaenus melanurus & $\mathrm{R}$ & $\mathrm{R}$ & $\mathrm{SB}, \mathrm{Vs}$ & \\
\hline Polioptila caerulea प्र & $\mathrm{R}$ & $\mathrm{C}$ & $\mathrm{SB}, \mathrm{Vs}, \mathrm{Et}, \mathrm{Cv}, \mathrm{Vr}, \mathrm{Us}$ & \\
\hline Polioptila albiloris $\bullet \mathbf{1 , 3}$ & $\mathrm{R}$ & & & \\
\hline Polioptila plumbea $\mathbf{v}$ & $\mathrm{R}$ & $\mathrm{E}$ & $\mathrm{SB}, \mathrm{ST}, \mathrm{Vs}, \mathrm{Et}, \mathrm{Cv}, \mathrm{Vr}$ & $\operatorname{Pr}$ \\
\hline Sialia sialis $\bullet \uparrow$ & $\mathrm{R}$ & & & \\
\hline Catharus aurantiirostris $\square \mathbf{3}$ & $\mathrm{R}$ & $\mathrm{R}$ & SB & \\
\hline Catharus frantzii $\bullet \uparrow$ & $\mathrm{R}$ & & & A \\
\hline Catharus mexicanus $\bullet \uparrow$ & $\mathrm{R}$ & & & $\operatorname{Pr}$ \\
\hline Catharus minimus $\mathbf{v}$ & $\mathrm{T}$ & $\mathrm{R}$ & SB & \\
\hline Catharus ustulatus ם & W & I & $\mathrm{SB}, \mathrm{ST}$ & \\
\hline Hylocichla mustelina $\mathbf{\square}$ & $\mathrm{T}$ & I & $\mathrm{ST}$ & \\
\hline Turdus grayi $\square \mathbf{1 , 3 \mathbf { 7 }}$ & $\mathrm{R}$ & $\mathrm{C}$ & $\mathrm{SB}, \mathrm{ST}, \mathrm{Vs}, \mathrm{Et}, \mathrm{Cv}, \mathrm{Vr}, \mathrm{Us}$ & \\
\hline Turdus assimilis $\mathbf{\bullet 1 , 3} \uparrow$ & $\mathrm{R}$ & & & \\
\hline Dumetella carolinensis $\square$ & W & I & $\mathrm{SB}, \mathrm{Vs}$ & \\
\hline Mimus gilvus ם & $\mathrm{R}$ & I & $\mathrm{Vs}, \mathrm{C}, \mathrm{Cv}$ & \\
\hline Melanotis hypoleucus $\bullet \uparrow$ & $\mathrm{R}$ & & $\mathrm{SB}, \mathrm{Vs}, \mathrm{C}, \mathrm{Cv}$ & \\
\hline Bombycilla cedrorum $\square \mathbf{v}$ & W & I & $\mathrm{SB}, \mathrm{Vs}, \mathrm{Et}, \mathrm{Cv}$ & \\
\hline Ptilogonys cinereus $\bullet 1$ & $\mathrm{R}$ & & & \\
\hline Vermivora pinus & W & $\mathrm{R}$ & $\mathrm{SB}, \mathrm{Vs}$ & \\
\hline Vermivora chrysoptera $\bullet$ & $\mathrm{W}$ & & & \\
\hline
\end{tabular}




\begin{tabular}{|c|c|c|c|c|}
\hline & $\begin{array}{c}\text { Estatus } \\
\text { migratorio }\end{array}$ & $\begin{array}{c}\text { Abundancia } \\
\text { relativa }\end{array}$ & $\begin{array}{c}\text { Tipos de } \\
\text { vegetación }\end{array}$ & $\begin{array}{c}\text { Estatus de } \\
\text { conservación }\end{array}$ \\
\hline Vermivora peregrina $\bullet$ & $\mathrm{W}$ & & & \\
\hline Vermivora celata $\mathbf{v}$ & W & $\mathrm{R}$ & SB & \\
\hline Vermivora ruficapilla $\square$ & W & $\mathrm{E}$ & $\mathrm{SB}, \mathrm{Vs}, \mathrm{Et}, \mathrm{Cv}, \mathrm{Vr}, \mathrm{Us}$ & \\
\hline Parula americana $\mathbf{\nabla}$ & W & I & $\mathrm{SB}, \mathrm{CV}$ & \\
\hline Parula pitiayumi $\mathbf{1 , 3 \mathbf { v } \uparrow}$ & $\mathrm{R}$ & I & $\mathrm{SB}, \mathrm{CV}$ & \\
\hline Dendroica petechia $\square \mathbf{3} \mathbf{v}$ & W & $\mathrm{E}$ & $\mathrm{SB}, \mathrm{Et}, \mathrm{Cv}, \mathrm{Vr}, \mathrm{Us}$ & \\
\hline Dendroica pensylvanica $\square$ \ & $\mathrm{W}$ & $\mathrm{R}$ & SB & \\
\hline Dendroica magnolia $\square \mathbf{v}$ & $\mathrm{W}$ & $\mathrm{E}$ & $\mathrm{SB}, \mathrm{ST}, \mathrm{Vs}, \mathrm{Et}, \mathrm{Cv}, \mathrm{Vr}, \mathrm{Us}$ & \\
\hline Dendroica coronata $\square \mathbf{v \downarrow}$ & $\mathrm{W}$ & $\mathrm{R}$ & ST & \\
\hline Dendroica virens $\square$ & W & $\mathrm{E}$ & $\mathrm{SB}, \mathrm{ST}, \mathrm{Vs}, \mathrm{Et}, \mathrm{Cv}, \mathrm{Vr}, \mathrm{Us}$ & \\
\hline Dendroica townsendi & W & $\mathrm{R}$ & $\mathrm{SB}, \mathrm{Cv}$ & \\
\hline Dendroica occidentalis $\square$ & W & I & $\mathrm{SB}, \mathrm{ST}, \mathrm{Vs}, \mathrm{Cv}$ & \\
\hline Dendroica fusca $\square \mathbf{v}$ & $\mathrm{T}$ & $\mathrm{R}$ & SB & \\
\hline Dendroica dominica $\bullet$ & W & & & \\
\hline Dendroica graciae $\square \mathbf{v}$ & $\mathrm{R}$ & $\mathrm{E}$ & $\mathrm{SB}, \mathrm{ST}, \mathrm{Et}, \mathrm{Cv}$ & \\
\hline Dendroica castanea $\bullet \uparrow$ & $\mathrm{T}$ & & & \\
\hline Mniotilta varia & W & $\mathrm{E}$ & $\mathrm{SB}, \mathrm{ST}, \mathrm{Vs}, \mathrm{Et}, \mathrm{Cv}, \mathrm{Us}$ & \\
\hline Protonotaria citrea $\mathbf{\imath}$ & $\mathrm{T}$ & $\mathrm{R}$ & SB & \\
\hline Helmitheros vermivorum $\square \mathbf{v}$ & W & $\mathrm{R}$ & ST & \\
\hline Seiurus aurocapilla $\square \mathbf{v}$ & W & $\mathrm{E}$ & $\mathrm{SB}, \mathrm{ST}, \mathrm{Vs}$ & \\
\hline Seiurus noveboracensis ㅁ & W & $\mathrm{R}$ & $\mathrm{SB}, \mathrm{ST}, \mathrm{Vs}$ & \\
\hline Seiurus motacilla $\bullet$ & W & & & \\
\hline Oporornis formosus ㅁ• & $\mathrm{T}$ & $\mathrm{R}$ & $\mathrm{SB}, \mathrm{ST}$ & \\
\hline Oporornis philadelphia $\bullet$ & $\mathrm{T}$ & & SB & \\
\hline Oporornis tolmiei $\square$ & W & I & SB & \\
\hline Geothlypis trichas $\square \mathbf{v}$ & $\mathrm{W}$ & $\mathrm{E}$ & $\mathrm{Pp}, \mathrm{C}$ & \\
\hline Geothlypis poliocephala $\square \mathbf{1} \mathbf{v}$ & $\mathrm{R}$ & $\mathrm{E}$ & $\mathrm{Pp}, \mathrm{C}$ & \\
\hline Wilsonia citrina $\square$ व $\uparrow$ & W & $\mathrm{R}$ & $\mathrm{SB}, \mathrm{Vs}, \mathrm{Cv}$ & \\
\hline Wilsonia pusilla $\square \mathbf{v}$ & W & $\mathrm{E}$ & $\mathrm{SB}, \mathrm{ST}, \mathrm{Vs}, \mathrm{Et}, \mathrm{Cv}, \mathrm{Vr}, \mathrm{Us}$ & \\
\hline Wilsonia canadensis $\square \mathbf{v}$ & $\mathrm{T}$ & I & $\mathrm{SB}, \mathrm{Vs}, \mathrm{Cv}$ & \\
\hline Myioborus pictus $\bullet \uparrow$ & $\mathrm{R}$ & & & \\
\hline Myioborus miniatus $\bullet \uparrow$ & $\mathrm{R}$ & & & \\
\hline Euthlypis lachrymosa $\mathbf{\square} \mathbf{1 , 3 \mathbf { v }}$ & $\mathrm{R}$ & I & $\mathrm{SB}, \mathrm{ST}, \mathrm{Vs}, \mathrm{Vr}$ & $\operatorname{Pr}$ \\
\hline Basileupterus culicivorus $\bullet$ & $\mathrm{R}$ & & & \\
\hline Basileupterus rufifrons $\mathbf{\square} \mathbf{1 , 3 \mathbf { v }}$ & $\mathrm{R}$ & I & $\mathrm{SB}, \mathrm{Vs}, \mathrm{Cv}$ & \\
\hline Icteria virens & W & I & Vs, Cv & \\
\hline
\end{tabular}




\begin{tabular}{|c|c|c|c|c|}
\hline & $\begin{array}{c}\text { Estatus } \\
\text { migratorio }\end{array}$ & $\begin{array}{c}\text { Abundancia } \\
\text { relativa }\end{array}$ & $\begin{array}{c}\text { Tipos de } \\
\text { vegetación }\end{array}$ & $\begin{array}{c}\text { Estatus de } \\
\text { conservación }\end{array}$ \\
\hline Chlorospingus ophthalmicus • & $\mathrm{R}$ & & & \\
\hline Eucometis penicillata $\mathbf{\uparrow}$ & $\mathrm{R}$ & $\mathrm{R}$ & $\mathrm{SB}, \mathrm{Vs}$ & \\
\hline Lanio aurantius $\bullet \uparrow$ & $\mathrm{R}$ & & & $\operatorname{Pr}$ \\
\hline Thraupis episcopus $\square \mathbf{\uparrow}$ & $\mathrm{R}$ & $\mathrm{R}$ & $\mathrm{Vs}, \mathrm{Cv}$ & \\
\hline Thraupis abbas 뮈 & $\mathrm{R}$ & $\mathrm{E}$ & $\mathrm{SB}, \mathrm{ST}, \mathrm{Et}, \mathrm{Cv}, \mathrm{Vr}$ & \\
\hline Cyanerpes cyaneus $\mathbf{v}$ & $\mathrm{R}$ & $\mathrm{R}$ & $\mathrm{SB}, \mathrm{ST}$ & \\
\hline Saltator coerulescens $\square \mathbf{1 , 3 \mathbf { v }}$ & $\mathrm{R}$ & I & $\mathrm{SB}, \mathrm{Vs}, \mathrm{Cv}$ & \\
\hline Saltator atriceps $\square \mathbf{1 \nabla}$ & $\mathrm{R}$ & $\mathrm{E}$ & $\mathrm{SB}, \mathrm{Vs}, \mathrm{Cv}$ & \\
\hline Volatinia jacarina $\square$ & $\mathrm{R}$ & $\mathrm{C}$ & $\mathrm{Pp}, \mathrm{C}$ & \\
\hline Sporophila torqueola $\square \mathbf{1 , 2} \mathbf{\nabla}$ & $\mathrm{R}$ & $\mathrm{E}$ & $\mathrm{Pp}, \mathrm{C}, \mathrm{Us}$ & \\
\hline Oryzoborus funereus $\mathbf{v}$ & $\mathrm{R}$ & $\mathrm{E}$ & $\mathrm{Pp}, \mathrm{C}$ & \\
\hline Tiaris olivaceus $\square \uparrow$ & $\mathrm{R}$ & $\mathrm{R}$ & $\mathrm{Vs}, \mathrm{Pp}, \mathrm{C}, \mathrm{Cv}$ & \\
\hline Arremon brunneinucha $\bullet$ & $\mathrm{R}$ & & & \\
\hline Arremonops rufivirgatus $\square^{3} \mathbf{}$ & $\mathrm{R}$ & $\mathrm{C}$ & $\mathrm{SB}, \mathrm{ST}, \mathrm{Vs}, \mathrm{Pp}, \mathrm{C}, \mathrm{Cv}, \mathrm{Us}$ & \\
\hline Aimophila botterii $\square$ & $\mathrm{R}$ & I & $\mathrm{Pp}, \mathrm{C}$ & \\
\hline Aimophila rufescens $\square \mathbf{v}$ & $\mathrm{R}$ & $\mathrm{E}$ & $\mathrm{Pp}, \mathrm{C}$ & \\
\hline Ammodramus savannarum $\bullet$ & W & & & \\
\hline Piranga flava $\bullet^{\diamond_{\uparrow}}$ & $\mathrm{R}$ & & & \\
\hline Piranga rubra $\square \mathbf{v}$ & W & I & $\mathrm{SB}, \mathrm{ST}, \mathrm{Vs}$ & \\
\hline Piranga ludoviciana $\square \mathbf{v}$ & W & E & $\mathrm{SB}, \mathrm{ST}, \mathrm{Vs}, \mathrm{Et}, \mathrm{Cv}$ & \\
\hline Piranga leucoptera $\bullet \uparrow$ & $\mathrm{R}$ & & & \\
\hline Habia rubica $\mathbf{\square} \mathbf{1 , 3} \mathbf{v}$ & $\mathrm{R}$ & I & $\mathrm{SB}, \mathrm{ST}, \mathrm{Vs}$ & \\
\hline Habia fuscicauda $\square$ & $\mathrm{R}$ & $\mathrm{R}$ & $\mathrm{SB}, \mathrm{ST}, \mathrm{Vs}$ & \\
\hline Pheucticus chrysopeplus $\mathbf{1} \mathbf{1 , 2 , 3 \mathbf { v }}$ & $\mathrm{R}$ & E & SB, ST, Vs, Et, Vr, Us & \\
\hline Pheucticus ludovicianus ם & $\mathrm{W}$ & $\mathrm{R}$ & Vs, Et & \\
\hline Granatellus venustus $\square \mathbf{1}, \mathbf{2}, 3^{\circ}{ }^{\uparrow}$ & $\mathrm{R}$ & I & $\mathrm{SB}, \mathrm{ST}, \mathrm{Vs}$ & \\
\hline Granatellus sallaei $\mathbf{1 , 3 \mathbf { v } \cdot \bullet}$ & $\mathrm{R}$ & $\mathrm{R}$ & $\mathrm{SB}, \mathrm{ST}, \mathrm{Vs}$ & \\
\hline Amaurospiza concolor $\bullet 1$ & $\mathrm{R}$ & & & \\
\hline Cyanocompsa parellina $\square \square^{1,3^{\circ}} \uparrow$ & $\mathrm{R}$ & $\mathrm{E}$ & $\mathrm{SB}, \mathrm{ST}, \mathrm{Vs}, \mathrm{C}$ & \\
\hline Passerina caerulea $\square$ & $\mathrm{R}$ & $\mathrm{C}$ & $\mathrm{Pp}, \mathrm{C}, \mathrm{Cv}$ & \\
\hline Passerina cyanea $\square \mathbf{v}$ & W & $\mathrm{E}$ & $\mathrm{SB}, \mathrm{Pp}, \mathrm{C}$ & \\
\hline Passerina versicolor $\square$ & $\mathrm{R}$ & $\mathrm{E}$ & $\mathrm{SB}, \mathrm{Vs}, \mathrm{Pp}, \mathrm{C}$ & \\
\hline Passerina ciris $\mathbf{\square}$ & W & I & Vs, C & \\
\hline Spiza americana $\bullet$ & $\mathrm{T}$ & & & \\
\hline Sturnella magna ${ }^{\circ}{ }_{\mathbf{v}}$ & $\mathrm{R}$ & $\mathrm{R}$ & $\mathrm{Pp}, \mathrm{C}$ & \\
\hline Dives dives $\square \mathbf{1} \mathbf{v}$ & $\mathrm{R}$ & $\mathrm{A}$ & $\mathrm{SB}, \mathrm{ST}, \mathrm{Vs}, \mathrm{Et}, \mathrm{Cv}, \mathrm{Vr}, \mathrm{Us}$ & \\
\hline
\end{tabular}




\begin{tabular}{|c|c|c|c|c|}
\hline & $\begin{array}{c}\text { Estatus } \\
\text { migratorio }\end{array}$ & $\begin{array}{c}\text { Abundancia } \\
\text { relativa }\end{array}$ & $\begin{array}{c}\text { Tipos de } \\
\text { vegetación }\end{array}$ & $\begin{array}{c}\text { Estatus de } \\
\text { conservación }\end{array}$ \\
\hline Quiscalus mexicanus $\square$ & $\mathrm{R}$ & SB & \multicolumn{2}{|c|}{$\mathrm{SB}, \mathrm{ST}, \mathrm{Vs}, \mathrm{Et}, \mathrm{Pp}, \mathrm{C}, \mathrm{Cv}, \mathrm{Vr}, \mathrm{Us}$} \\
\hline Molothrus aeneus $\square \mathbf{3} \mathbf{v}$ & $\mathrm{R}$ & $\mathrm{C}$ & \multicolumn{2}{|c|}{ Vs, Et, Pp, C, Cv, Vr, Us } \\
\hline Icterus prosthemelas $\square \mathbf{1} \mathbf{\uparrow}$ & $\mathrm{R}$ & $\mathrm{R}$ & \multicolumn{2}{|l|}{$\mathrm{SB}, \mathrm{Vs}, \mathrm{Cv}$} \\
\hline Icterus wagleri $\square \mathbf{1}, \mathbf{3} \mathbf{v}$ & $\mathrm{R}$ & $\mathrm{E}$ & \multicolumn{2}{|l|}{$\mathrm{SB}, \mathrm{Vs}, \mathrm{Cv}$} \\
\hline Icterus maculialatus $\square \mathbf{1} \mathbf{v}$ & $\mathrm{R}$ & I & SB & $\operatorname{Pr}$ \\
\hline Icterus spurius ם & W & $\mathrm{R}$ & \multicolumn{2}{|l|}{$\mathrm{Vs}, \mathrm{C}, \mathrm{Cv}$} \\
\hline Icterus chrysater $\bullet \mathbf{3}$ & $\mathrm{R}$ & & & \\
\hline Icterus mesomelas ${ }^{3 \mathbf{v} \uparrow}$ & $\mathrm{R}$ & I & \multicolumn{2}{|l|}{$\mathrm{SB}, \mathrm{Vs}, \mathrm{Cv}$} \\
\hline Icterus pustulatus $\square \mathbf{1 , 2 , 3} \mathbf{v}$ & $\mathrm{R}$ & $\mathrm{C}$ & \multicolumn{2}{|c|}{$\mathrm{SB}, \mathrm{ST}, \mathrm{Vs}, \mathrm{Et}, \mathrm{Cv}, \mathrm{Vr}, \mathrm{Us}$} \\
\hline Icterus gularis $\square \mathbf{1 , 2} \mathbf{2}$ & $\mathrm{R}$ & $\mathrm{C}$ & \multicolumn{2}{|c|}{$\mathrm{SB}, \mathrm{ST}, \mathrm{Vs}, \mathrm{Cv}, \mathrm{Vr}, \mathrm{Us}$} \\
\hline Icterus galbula $\square \mathbf{v}$ & W & I & \multicolumn{2}{|c|}{$\mathrm{SB}, \mathrm{Cv}, \mathrm{Vr}, \mathrm{Us}$} \\
\hline Amblycercus holosericeus & $\mathrm{R}$ & I & \multicolumn{2}{|l|}{$\mathrm{SB}, \mathrm{ST}$} \\
\hline Cacicus melanicterus $\mathbf{1} \mathbf{1 , 2 \nabla}$ & $\mathrm{T}$ & $\mathrm{R}$ & \multicolumn{2}{|l|}{ SB } \\
\hline Psarocolius wagleri & $\mathrm{R}$ & $\mathrm{R}$ & $\mathrm{SB}, \mathrm{ST}$ & $\operatorname{Pr}$ \\
\hline Psarocolius montezuma $\square \mathbf{1} \mathbf{v} \uparrow$ & $\mathrm{R}$ & $\mathrm{R}$ & $\mathrm{SB}, \mathrm{Vs}$ & $\operatorname{Pr}$ \\
\hline Euphonia affinis $\mathbf{\square} \mathbf{1 , 2 \nabla \bullet}$ & $\mathrm{R}$ & $\mathrm{E}$ & \multicolumn{2}{|c|}{$\mathrm{SB}, \mathrm{Vs}, \mathrm{Cv}, \mathrm{Vr}, \mathrm{Us}$} \\
\hline Euphonia hirundinacea $\square \mathbf{1 , 2 \nabla}$ & $\mathrm{R}$ & $\mathrm{E}$ & \multicolumn{2}{|c|}{$\mathrm{SB}, \mathrm{ST}, \mathrm{Vs}, \mathrm{Et}, \mathrm{Cv}, \mathrm{Vr}$} \\
\hline Euphonia elegantissima $\square \mathbf{1 \mathbf { v }}$ & $\mathrm{R}$ & $\mathrm{R}$ & \multicolumn{2}{|c|}{$\mathrm{SB}, \mathrm{ST}, \mathrm{Vs}, \mathrm{Et}, \mathrm{Cv}$} \\
\hline Carpodacus mexicanus $\square \uparrow$ & $\mathrm{R}$ & $\mathrm{R}$ & \multicolumn{2}{|l|}{$\mathrm{Pp}, \mathrm{C}, \mathrm{Us}$} \\
\hline Carduelis psaltria $\square$ & $\mathrm{R}$ & $\mathrm{E}$ & \multicolumn{2}{|c|}{ Vs, Et, Pp, C, Cv, Vr, Us } \\
\hline Passer domesticus $\square \mathbf{v}$ & $\mathrm{R}$ & $\mathrm{E}$ & \multicolumn{2}{|l|}{ Us } \\
\hline
\end{tabular}

